\title{
1 Membrane binding controls ordered self-assembly of animal septins
}

2

3

4

5

Agata Szuba ${ }^{1 \#}$, Fouzia Bano ${ }^{2 \$}$, François Iv ${ }^{3}$, Manos Mavrakis ${ }^{3 *}$, Ralf P. Richter ${ }^{2 *}$, Aurelie Bertin ${ }^{4,5^{*}}$, Gijsje H. Koenderink ${ }^{1,6^{*}}$

${ }^{1}$ AMOLF, Department of Living Matter, Biological Soft Matter group, Science Park 104, 1098 XG Amsterdam, The Netherlands

${ }^{2}$ School of Biomedical Sciences, Faculty of Biological Sciences, School of Physics and Astronomy, Faculty of Engineering and Physical Sciences, Astbury Centre for Structural Molecular Biology, and Bragg Centre for Materials Research, University of Leeds, Leeds, LS2 9JT, United Kingdom

${ }^{3}$ Institut Fresnel, CNRS, Aix-Marseille Univ, Centrale Marseille, 13013 Marseille, France

${ }^{4}$ Laboratoire Physico Chimie Curie, Institut Curie, PSL Research University, CNRS UMR168, 75005, Paris, France

${ }^{5}$ Sorbonne Université, 75005 , Paris

${ }^{6}$ Department of Bionanoscience, Kavli Institute of Nanoscience Delft, Delft University of Technology, Van der Maasweg 9, 2629 HZ Delft, The Netherlands

*Corresponding authors: g.h.koenderink@tudelft.nl, $\underline{\text { r.richter@leeds.ac.uk, manos.mavrakis@univ-amu.fr, }}$ aurelie.bertin@curie.fr

"Current address: Pollard Institute, School of Electronic \& Electrical Engineering, University of Leeds, Leeds, LS2 9JT, United Kingdom

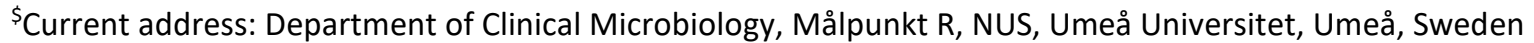

\section{Abstract}

Septins are conserved cytoskeletal proteins that regulate cell cortex mechanics. The mechanisms of their interactions with the plasma membrane remain poorly understood. Here we show by cell-free reconstitution that membrane binding requires electrostatic interactions of septins with anionic lipids and promotes the ordered self-assembly of fly septins into filamentous meshworks. Transmission electron microscopy reveals that both fly and mammalian septins form arrays of single and paired filaments. Atomic force microscopy and quartz crystal microbalance demonstrate that the fly filaments form mechanically rigid, 12 to $18 \mathrm{~nm}$ thick, double layers of septins. By contrast, C-terminally truncated septin mutants form $4 \mathrm{~nm}$ thin monolayers, indicating that stacking requires the C-terminal coiled coils on DSep2 and Pnut subunits. Our work shows that membrane binding is required for fly septins to form ordered arrays of single and paired filaments and provides new insights into the mechanisms by which septins may regulate cell surface mechanics. 
Membrane binding controls ordered self-assembly of animal septins

\section{Introduction}

Septins are a conserved family of cytoskeletal proteins [1] capable of forming filamentous scaffolds at the cell cortex that participate in many processes such as cytokinesis, cell-cell adhesion, and phagocytosis [25]. Most of what we currently know about the molecular mechanisms by which septins function comes from studies of the budding yeast cell S. cerevisae, where septins form hetero-octamers [6-10] that form paired filaments [11-13]. During budding, septins form a collar encircling the bud neck that acts as a scaffold to recruit proteins necessary for cell division [14-16], and that restricts lateral diffusion of transmembrane proteins [17-19]. Compared to yeast septins, animal septins have a much more variable cellular localization that changes with tissue type, developmental stage, and cell cycle state [20, 21]. They are frequently found at curved regions of the plasma membrane such as the base of dendritic spines and cilia, where they provide a diffusion barrier [22-24]. However, animal septins also associate with flat regions of the cell cortex, where they influence the rigidity and contractility of the actin-myosin cortex within single cells [25-27] and within multicellular tissues [28-30]. Cortical septins also play a key role in cell division, where they are needed to stabilize the actomyosin ring [31] and recruit proteins that mediate chromosome segregation and abscission [32-34].

It remains unclear whether the cortical septin pool in animal cells associates with the plasma membrane itself or with membrane-bound actin filaments and microtubules [35, 36]. Biochemical reconstitution studies have shown that mammalian septins can bind phosphoinositides [4, 37-40], but septins have also been shown to bind actin filaments [41-43] and various actin-binding proteins including anillin [44] and non-muscle myosin-2 [45]. Electron microscopy of immuno-stained cells revealed localization of cortical septins with cortical actin in tissue culture cells $[36,46]$, but the high density of the actin cortex in animal cells has made it impossible to determine whether cortical septins directly interact with the plasma membrane. It is even unclear whether cortical septins truly form filaments. Fluorescence microscopy has shown the presence of cortical septin puncta, fibrous strands, or rings [25-27, 29], but has lacked the resolution to resolve the precise nature of these structures. Septin-deficient cells exhibit a markedly reduced cortical rigidity [25-27, 29], but it is unclear whether these effects imply a loss of membrane-bound septin filaments or a loss of septin-mediated actin stabilization. A further complication is that microtubules have also been found to interact with cortical septins [47].

To resolve the functions of septins at the animal cell cortex, it is necessary to understand the innate ability of septins to assemble alone and in association with the membrane. Several reconstitution studies have been reported for native and recombinant septins from various animal species, but nearly all of these studies considered septin assembly in bulk solution. Frog (Xenopus laevis) septins were found to form paired filaments similar to yeast septins [48], while recombinant nematode (C. Elegans) septins [49] and native and recombinant fly (Drosophila melanogaster) septins [41, 50, 51] were observed to form bundles. Recombinant mammalian septin hexamers have been shown to form rings and spirals that are reminiscent of structures formed by Shs1-capped yeast septin octamers [52]. To the best of our knowledge, there are only two reports until now of the organization of animal septins on lipid membranes, showing that septins in porcine brain extracts can tubulate giant liposomes and form filamentous meshworks encasing the membrane tubes [38, 40].

Here we investigate the role of membrane-binding in septin assembly by reconstituting recombinant animal septins on flat supported lipid bilayers. We focus on septin hexamers composed of Pnut, DSep2, and DSep1 from the model organism Drosophila. We studied the septins on model cell membranes composed of phosphatidylcholine (PC) lipids combined with either phosphatidylserine (PS) or 
Membrane binding controls ordered self-assembly of animal septins

phosphatidylinositol(4,5)-bisphosphate (hereafter referred to as $\left.\mathrm{PI}(4,5) \mathrm{P}_{2}\right)$. PS is the most abundant anionic lipid in the inner leaflet of the plasma membrane [53]. The cell membrane in the fly has been reported to contain 3-10\% PS, but the composition varies among tissues and developmental stages [5456]. $\mathrm{PI}(4,5) \mathrm{P}_{2}$ is an anionic signaling lipid previously shown to interact with yeast and mammalian septins $[37,57,58]$ and assumed to be important for septin-membrane interactions in the fly $[59,60]$. We show by fluorescence imaging that fly septin hexamers have a high affinity for membranes containing PS and/or $\mathrm{PI}(4,5) \mathrm{P}_{2}$, and form dense membrane-associated meshworks. Nanometer-resolution imaging by electron microscopy and by atomic force microscopy reveals that these meshworks comprise single and paired filaments, which laterally associate into bundles that form tightly packed domains. We propose a molecular model of the septin self-assembly mechanism that is consistent with these new data. Our findings establish that animal septins have the innate ability to form stable filamentous meshworks on lipid membranes, which likely contributes to their ability to regulate cell cortex rigidity and tension.

\section{Results}

\section{Septins form bundles in solution}

To understand how membranes influence septin assembly, we begin by analyzing how septins assemble in free solution. We focus on septin hexamers composed of DSep1, DSep2, and Pnut from the model organism Drosophila, which have been previously characterized in vivo [41,61-63] and in vitro [41, 50, 51 ], and which are highly homologous to their human septin orthologs (DSep1, DSep2 and Pnut are 75\%, $68 \%$ and 65\% identical to Sept2, Sept6 and Sept7, respectively; see Methods section). Based on phylogenetic analysis that classifies fly and human septins in distinct subgroups (DSep1/Sept2 in the SEPT2 subgroup; DSep2/Sept6 in the SEPT6 subgroup; Pnut/Sept7 in the SEPT7 subgroup [64, 65]), and the order of subunits in mammalian septin complexes $[66,67]$, fly septin hexamers should have a palindromic arrangement with the Pnut subunits in the center, the DSep1 subunits at the termini, and the DSep2 subunits in between (Figure 1C). Each subunit carries a flexible carboxy-terminal extension, a part of which is predicted to adopt a coiled-coil arrangement [68]. Using the coiled-coil prediction algorithm COILS (see Methods section), we predict the C-terminus of DSep1 to form a coiled-coil that is 28 residues long, and the C-termini of Pnut and DSep2 to form coiled-coils that are 86 residues long. Our coiled-coil prediction analysis for human septins yields the same predictions, i.e., 28-residue-long coiled-coils for Sept2 and 86residue-long coiled-coils for each Sept6 and Sept7 (Figure 1B). Considering a $1.5 \AA$ Aise per residue in an alpha-helix, we estimate the coiled-coil of DSep1/Sept2 to be $4 \mathrm{~nm}$ long, and the coiled-coils of DSep2/Sept6 and Pnut/Sept7 to be $13 \mathrm{~nm}$ long (Figure 1C). Hexamers form $24 \mathrm{~nm}$-long rods, with the globular domains of the septin subunits approximately $4 \mathrm{~nm}$ in diameter $[41,69]$. The coiled-coil of DSep1 could thus extend as much as the G-domain itself, while the coiled-coils of DSep2 and Pnut could even extend as much as 3 -fold the size of the G-domain. From the end of the $\alpha 6$-helix to the start of the predicted coiled-coils, there are stretches of 24, 15 and 15 residues for DSep1, DSep2 and Pnut, respectively (see Figure $\mathbf{1 A}$ ), that are predicted to be unstructured. Given a rise per residue of $\sim 0.4 \mathrm{~nm}$, these lengths translate to contour lengths of 6 to $10 \mathrm{~nm}$. These regions thus likely act as a flexible hinge between the G-domain and the coiled-coil, allowing the coiled-coils to pivot around their anchor points. This prediction is consistent with observations that coiled coils did not show up in X-ray crystal structures or particle-averaged electron microscopy (EM) analysis of yeast and mammalian septin oligomers $[9,69]$. 
Membrane binding controls ordered self-assembly of animal septins

Moreover, EM images of yeast, Drosophila, and C. elegans septin oligomers showed direct evidence that the coiled coils can sweep out a large volume $[9,41,49]$.

To test the ability of the recombinant fly septins to polymerize in bulk solution, we performed TIRF imaging of mEGFP-tagged septin hexamers (Figure 1D) after rapid dilution from a high salt storage buffer containing $300 \mathrm{mM} \mathrm{KCl}$ to a low salt polymerization buffer containing $50 \mathrm{mM} \mathrm{KCl}$. We expect fly septins to form bundles under these conditions $[41,70]$. To enable observation of septins in the thin (100 $\mathrm{nm}$ ) evanescent TIRF field, we pushed them down onto a coverslip passivated with a neutral (PC) lipid bilayer with the crowding agent methylcellulose. As shown in Figure 1D, septins did not form any structures visible at the resolution of the light microscope until the concentration reached $200 \mathrm{nM}$. When we increased the concentration further to $500 \mathrm{nM}$, the septins formed longer bundles. Bundle formation was very rapid, taking less than 2 minutes for completion. The bundles were rather straight and did not exhibit measurable thermal bending undulations, indicating that they must be stiff.

To measure the width and mass of the septin bundles, we turned to scanning transmission electron microscopy, which provides quantitative mass measurements based on the dark-field signal from elastically scattered electrons emitted under a high angle [71]. The sample is raster-scanned with a focused electron beam and the intensity in each image pixel is converted to the projected specimen mass by including tobacco mosaic virus (TMV) rods with a well-defined mass-per-length (MPL) of $131 \mathrm{kDa} / \mathrm{nm}$ as an internal calibration. To test the accuracy of the mass mapping method, we first imaged budding yeast septins, since these are already known to form paired filaments with a theoretical mass per length of $23.2 \mathrm{kDa} / \mathrm{nm}$ [9]. As shown in Figure 1E, the yeast septins form thin semiflexible polymers that are weaker in intensity than the TMV rods, consistent with their smaller mass per length. The average massper-length (based on 10 septin filaments from 3 images) was $\sim 20 \mathrm{kDa} / \mathrm{nm}$, close to the mass-per-length expected for paired filaments. In rare cases the image clearly showed a double-stranded structure with two filaments running in parallel with a small spacing in the range of $17 \mathrm{~nm}-27 \mathrm{~nm}$, consistent with prior transmission EM findings [9] (see lower panel of Figure 1E). By contrast, fly septins formed thick bundles that were stronger in intensity than the TMV rods, indicating a larger MPL (Figure 1F). Bundles formed at $200 \mathrm{nM}$ had tapered ends and a thicker center. The example bundle in Figure $1 \mathrm{~F}$ has a MPL of around 250 $\mathrm{kDa} / \mathrm{nm}$ in the center (marked by an asterisk) and a corresponding center width of $31 \mathrm{~nm}$. Given a calculated MPL of $12.8 \mathrm{kDa} / \mathrm{nm}$ per fly septin hexamer, this number translates to around 20 hexamers per cross-section. Considering that the inter-filament spacing has to be at least $4 \mathrm{~nm}$, a width of $31 \mathrm{~nm}$ and 20 hexamers per cross-section implies there are at least 3 monolayers. At $500 \mathrm{nM}$, the septin bundles were thicker (Figure 1G) with maximal widths of up to $280 \mathrm{~nm}$ and MPL values up to $\sim 5000 \mathrm{kDa} / \mathrm{nm}$ (Figure 1 - Figure Supplement 2), corresponding to $>400$ hexamers per cross-section or a thickness of at least 6 monolayers. This type of analysis suggests that septin bundles in solution do not grow as flat sheets but as 3D bundles. 

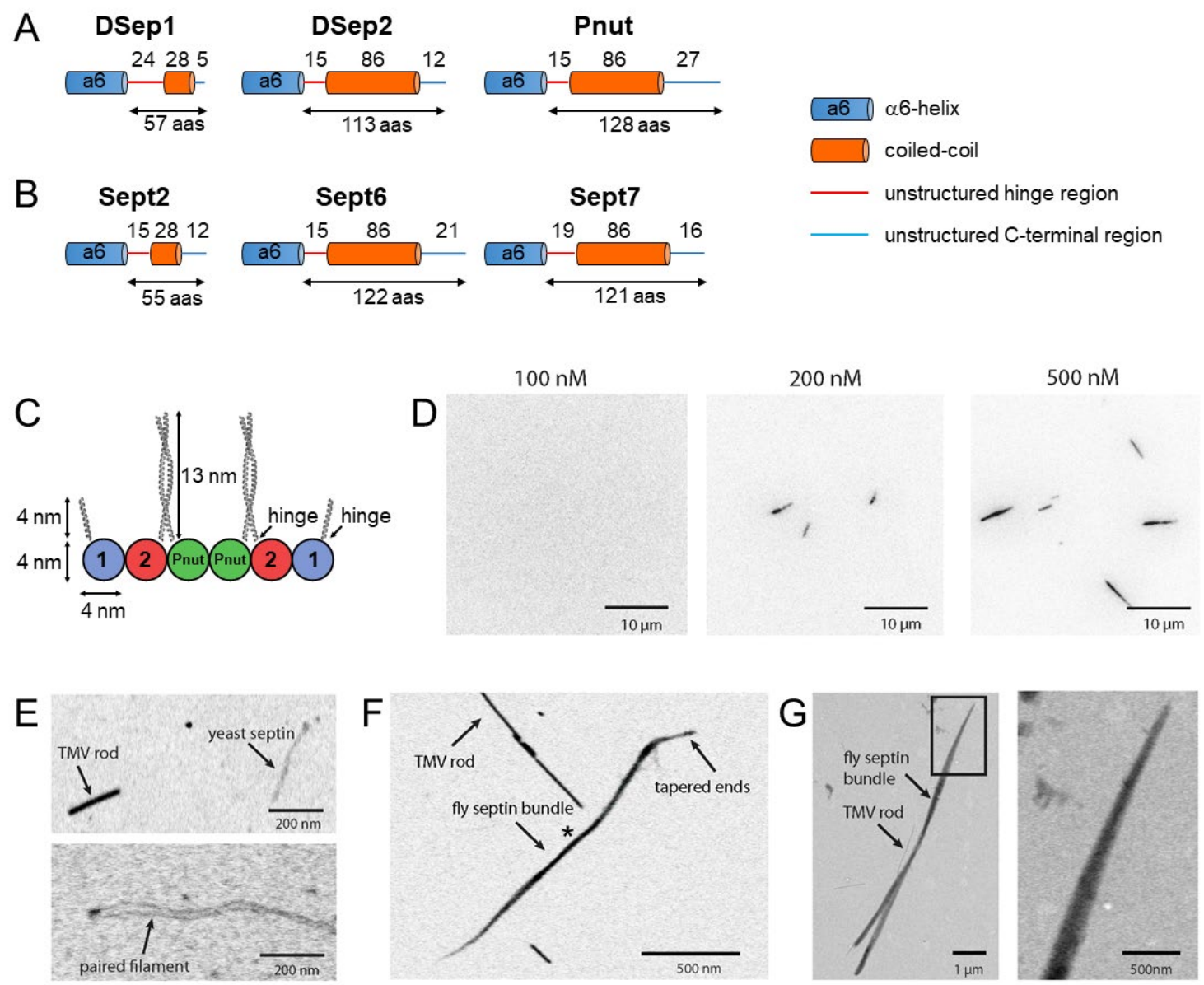

Figure 1: Fly septin hexamers form bundles in solution. (A) Structure predictions for the C-terminal regions of fly septins, starting from the end of the $\alpha 6$-helix regions. (B) Corresponding predictions for human septins. (C) Schematic of the fly septin hexamer, showing its expected palindromic arrangement with long (13 nm) coiled-coil extensions of the Pnut and DSep2 (2) subunits, and shorter ( $4 \mathrm{~nm}$ ) extensions on the DSep1 (1) subunits. The predicted septin coiled-coils are depicted to scale using available crystalstructures of coiled-coils (see Methods). (D) TIRF images of mEGFP-tagged fly septin bundles formed in solution and crowded onto net-neutral SLBs composed of PC by methylcellulose, with septin concentrations as indicated above each image. (E) STEM images of yeast septin filaments. Upper panel: septin filament and a TMV rod (see arrows). Lower panel: septin filament showing a paired arrangement of two linear strands. (F) STEM image of fly septin bundle formed at a concentration of $200 \mathrm{nM}$ surrounded by several TMV rods (one example is pointed out). The bundle center width and MPL (asterisk) are $31 \mathrm{~nm}$ and $250 \mathrm{kDa} / \mathrm{nm}$, respectively. (G) STEM image of a fly septin bundle formed at a concentration of 500 $\mathrm{nM}$, together with three TMV rods (one is indicated by an arrow). Note that TIRF and STEM images are all contrast-inverted, so septins appear dark against a light background.

The following figure supplements are available for figure 1:

Figure 1 - Figure supplement 1. Biochemical and morphological characterization of septins.

Figure 1 - Figure supplement 2. Quantification of the bundle size for septin bundles formed in bulk solution at $500 \mathrm{nM}$. 
Membrane binding controls ordered self-assembly of animal septins

\section{Lipid membranes recruit septins and promote their assembly}

To investigate how membrane-binding affects septin polymerization, we deposited the purified fly septin hexamers in polymerization buffer on glass-supported lipid bilayers (SLBs) composed of net-neutral PC lipids doped with anionic lipids (Figure $2 A$ ). To test whether fly septins bind $\mathrm{PI}(4,5) \mathrm{P}_{2}$, as reported for yeast septins $[57,58]$, we doped the $\mathrm{PC}$ membranes with different mole percentages of $\mathrm{PI}(4,5) \mathrm{P}_{2}$ ranging from 1 to $8 \%$. Already at $1 \% \mathrm{PI}(4,5) \mathrm{P}_{2}$, septins formed immobile bundles adhering to the membrane (Figure $2 \mathbf{B}$ ). We note that membrane recruitment was observed here in the absence of methylcellulose, showing that septins indeed bind to $\mathrm{PI}(4,5) \mathrm{P}_{2}$ lipids. As we raised the $\mathrm{PI}(4,5) \mathrm{P}_{2}$ content from $1 \%$ to $8 \%$, we observed a striking transition from a sparse arrangement of thick bundles to a dense protein layer. At intermediate $\mathrm{PI}(4,5) \mathrm{P}_{2}$ fractions of $2.5 \%$ and $5 \%$ we could still discern septin bundles, but at $8 \% \mathrm{PI}(4,5) \mathrm{P}_{2}$ the protein density was too high to discern any details of the ultrastructure.

To test whether fly septins selectively bind the $\mathrm{PI}(4,5) \mathrm{P}_{2}$ head group as reported for yeast septins $[57,58]$ or simply bind through nonspecific electrostatic interactions, we next replaced $\mathrm{PI}(4,5) \mathrm{P}_{2}$ by PS. As shown in Figure $\mathbf{2 C}$, fly septins were also recruited to the PS-containing bilayers and again showed a transition from a sparse distribution of thick bundles at low PS content to a dense layer at high PS content. The adsorbed amount of septins, as quantified from the total fluorescence intensity, linearly increased as the PS content was raised from $5 \%$ to $20 \%$ (Figure $\mathbf{2}$ - figure supplement $\mathbf{1 A}, \boldsymbol{B}$ ). The observation that PS has a qualitatively similar effect compared to $\mathrm{PI}(4,5) \mathrm{P}_{2}$ on septin recruitment and assembly on SLBs suggests that the membrane-binding affinity of fly septins is governed by the net surface charge of the membrane rather than by any specific affinity for $\mathrm{PI}(4,5) \mathrm{P}_{2}$. The transition from dilute septin bundles to

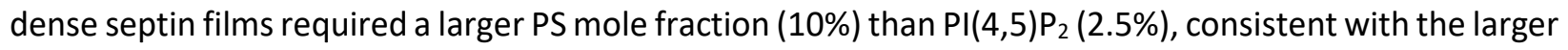
net charge (-4) of the head group of $\mathrm{PI}(4,5) \mathrm{P}_{2}$ due to the phosphate groups compared to the net charge (1 ) on the head group of PS [72-75]. Therefore, our findings suggest that the total negative surface charge of the membrane governs septin adsorption, indicating that the septin-membrane interaction is primarily electrostatic in origin.

In all cases, filamentous septin structures were already present as soon as we could start imaging ( 3 min after septin injection) and the structures did not change in number or thickness over time (Figure 2 - figure supplement $\mathbf{1 A}$ ). Apparently, septins bind strongly and rapidly to the membrane. Fluorescence recovery after photobleaching experiments showed that the septins exhibited negligible subunit exchange (Figure 2 - figure supplement $2 A$ ), indicating that they are indeed stably anchored to the membrane. The membrane underneath the septin film was nevertheless fluid, as indicated by FRAP measurements of rhodamine-labeled PE tracer lipids (Figure 2 - figure supplement $2 B$ ).

To test the dependence of septin adsorption on bulk septin concentration, we incubated bilayers containing 20 mole-\% PS with solutions of septin hexamers at concentrations ranging between 10 and 500 $\mathrm{nM}$. As shown in Figure 2D, we observed uniform septin layers with a texture that could not be resolved at the resolution of the TIRF microscope over this entire concentration range. The septin fluorescence intensity increased roughly linearly with septin concentration. Interestingly, septins already formed membrane-bound layers at $10 \mathrm{nM}$, i.e., at concentrations far below the onset concentration of $200 \mathrm{nM}$ for bundle formation in bulk solution (Figure 1B,C), suggesting that membrane association catalyzes polymerization. However, at the diffraction-limited resolution provided by TIRF microscopy we cannot distinguish whether the membrane-bound layers truly are filamentous. At concentrations above $200 \mathrm{nM}$, we observed the presence of septin bundles in the solution above the bilayer by confocal imaging, consistent with the observed onset for bundling in the absence of an adhesive membrane (Figure 1D). 
A

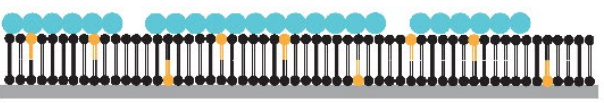

anionic lipid neutral lipid eopeses septin hexamer

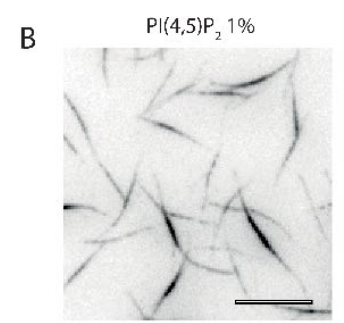

C
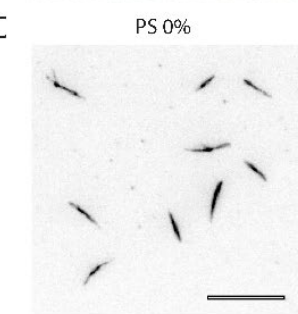

D
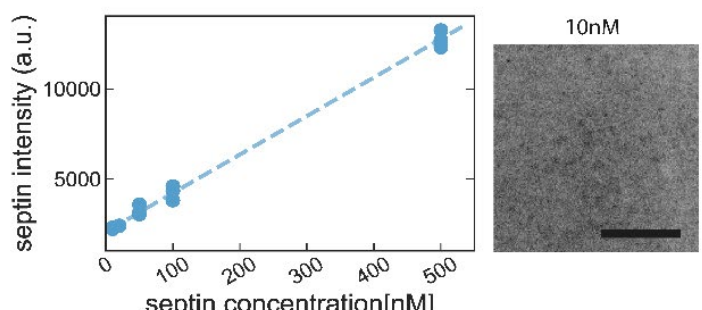

$\mathrm{PI}(4,5) \mathrm{P}_{2} 5 \%$

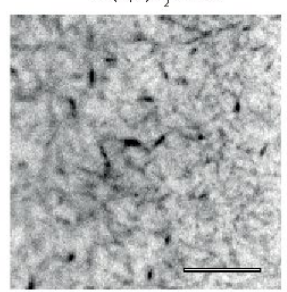

PS $5 \%$

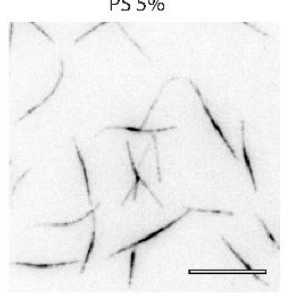

septin concentration[nM]

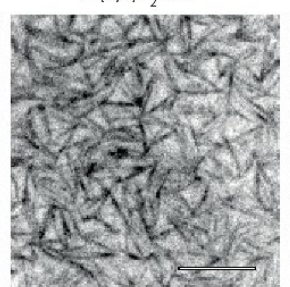

PS $10 \%$

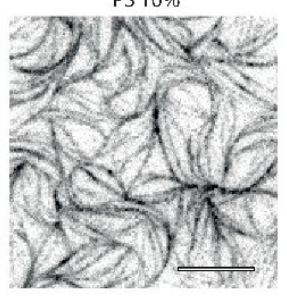

$\mathrm{PI}(4,5) \mathrm{P}_{2} 8 \%$

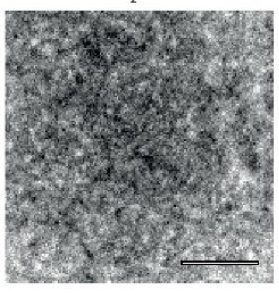

PS 20\%

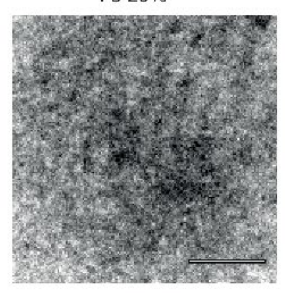

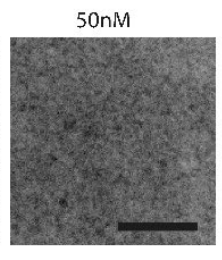

$500 \mathrm{nM}$

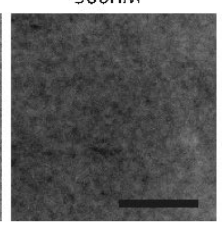

Figure 2: Glass-supported lipid bilayers containing anionic lipids recruit septins and promote the assembly of dense septin meshworks. (A) Purified fly septin hexamers are deposited on a glass-supported bilayer composed of net-neutral (PC) and anionic (PS or PI $(4,5) \mathrm{P}_{2}$ ) lipids (sketch not to scale). (B) TIRF images recorded $\sim 3$ min after the deposition of mEGFP-tagged septin hexamers $(1 \mu \mathrm{M})$ on bilayers doped with $\mathrm{PI}(4,5) \mathrm{P}_{2}$ at mole fractions between 1 and $8 \%$ (see legend). (C) TIRF images obtained $\sim 3$ min after the deposition of septins $(1 \mu \mathrm{M})$ on bilayers doped with PS at mole fractions between 5 and $20 \%$ (see legend). (D) Septins form dense layers on PC bilayers containing 20\% PS whose fluorescence intensity increases linearly with the solution concentration. Note that the imaging settings in panel (D) are different from those in panels $B, C$, so the intensities cannot be directly compared. The dashed line shows a linear fit to the data; the non-zero intercept reflects background fluorescence. Each data point is collected from 1 experiment with 3 different regions of interest in different locations. Note that all images are contrastinverted, so septins are dark and membrane areas devoid of septins are light. Scale bars: $10 \mu \mathrm{m}$.

The following figure supplements are available for figure 2:

Figure 2 - Figure supplement 1. Dependence of septin adsorption on the PS content of the SLBs.

Figure $\mathbf{2}$ - Figure supplement 2. FRAP data testing the mobility of septins and lipids. 
Membrane binding controls ordered self-assembly of animal septins

To discern the ultrastructure of membrane-adsorbed septins, we turned to transmission electron microscopy. We incubated septins with lipid monolayers, deposited these on EM grids, and negatively stained the protein with $2 \%$ uranyl formate, following a protocol previously used with yeast septins [57]. To test the role of membrane composition, we formed lipid monolayers by mixing PC lipids with either 20 mol-\% PS, 5 mol-\% PI $(4,5) \mathrm{P}_{2}$, or a combination of both that mimics the co-existence of both lipids in the plasma membrane of cells $[76,77]$. As shown in Figure $3 A-C$, septins formed densely packed arrays of thin filaments in all three cases, which is qualitatively consistent with the dense meshworks observed by TIRF microscopy. Close inspection of the EM micrographs revealed paired septin filaments (black arrows point out examples). Line profiles revealed an average center-to-center spacing of $5.6 \pm 0.7 \mathrm{~nm}(N=57$; see examples in Figure 3D). Given a width of $4 \mathrm{~nm}$ for fly septin hexamers [41], the spacing between the two filaments forming a pair is only $1.6 \mathrm{~nm}$. Pairing of yeast septin filaments has been reported to occur through antiparallel interactions of coiled coils on opposing filaments [9]. To accommodate the narrow spacing we observe, the longer $(\sim 13 \mathrm{~nm})$ coiled coils of the Pnut and DSep2 subunits (Figure 1C) of adjacent filaments may arrange anti-parallel and lie flat (i.e. parallel to the lipid plane), or they may arrange parallel and pointing upwards (i.e., away from the lipid plane).

On monolayers containing $20 \%$ PS plus $5 \% \mathrm{PI}(4,5) \mathrm{P}_{2}$, we could observe clear examples of paired filaments that were bridged at intervals of $24 \mathrm{~nm}$ by single hexamer rods recognizable by their $24 \mathrm{~nm}$ length (Figure $3 \mathbf{C}$ ), forming a filamentous network. These bridges were either perpendicular to the filaments they bridged (blue arrowheads) or under an angle (red arrowheads). We observed comparable arrays of tightly paired filaments connected by orthogonally or diagonally oriented single filaments for mammalian septins on lipid monolayers of the same composition (Figure 3-figure supplement 1). Similar arrays of filaments were also observed for budding yeast septins [52,57], suggesting that this architectural feature is conserved within eukaryotes. We do note a subtle difference for the mammalian septins as compared to the fly septins. For the fly septins, the bridges between paired filaments are single isolated hexamer rods. Instead, for the mammalian septins, the paired filaments (white arrows in Figure 3-figure supplement 1) are intersected with a distinct set of thinner continuous filaments (red arrows), creating a network of interconnected and perpendicular filaments. It is unclear whether the bridging filaments are single or paired; if paired, the filaments could be rotated or twisted to appear thinner.

Since the sample preparation for the TEM experiments requires drying and negative staining, we complemented these findings by cryo-EM imaging on large unilamellar vesicles comprised of PC and 6 mol-\% $\mathrm{PI}(4,5) \mathrm{P}_{2}$ incubated with septin hexamers. As shown in Figure 4, the cryoEM images confirm the presence of paired septin filaments on $\mathrm{PI}(4,5) \mathrm{P}_{2}$-containing lipid membranes (examples are traced out by double red lines in Figure $4 A, B$ ). The average center-to-center distance between the filaments forming a pair was $5.7 \pm 0.8 \mathrm{~nm}(N=40)$, in excellent agreement with the negative stain images of septin filaments on lipid monolayers (see Figure 4 - figure supplement 1 ). In addition, we occasionally observed single filaments or hexamers (examples are traced out by single red lines in Figure $4 \mathbf{B}$ ) including some that appeared to interconnect filaments at an orthogonal or oblique angle (blue lines in Figure $4 \mathbf{B}$ ). The density of filaments varied among vesicles (see Figure $\mathbf{4}$ and Figure $\mathbf{4}$ - figure supplement $\mathbf{2}$ ), which might be due to some variability in the proportion of charged lipids incorporated within the vesicles. Besides, as demonstrated previously $[78,79]$, the curvature of the vesicles affects the septin-vesicle interaction and most likely introduces additional variability in the density of filaments interacting with vesicles. Vesicles decorated with septin filaments typically appeared to have a faceted contour, whereas vesicles alone 
(A)
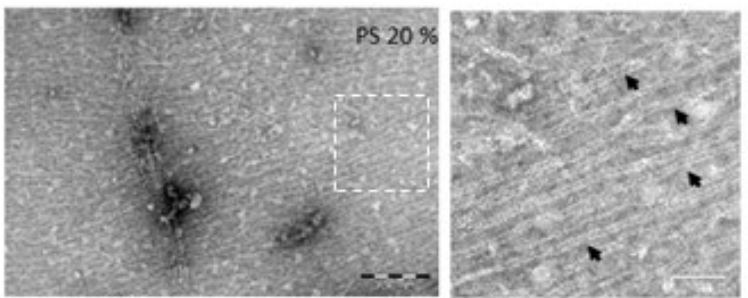

(B)
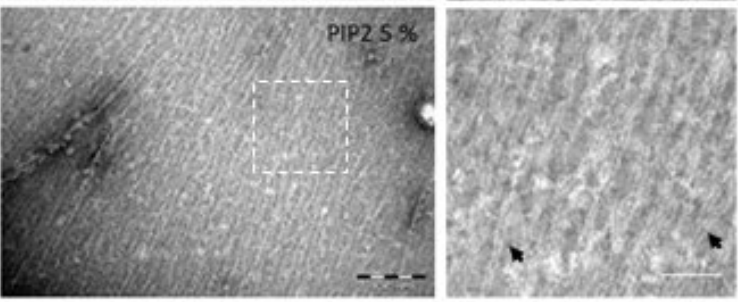

(C)
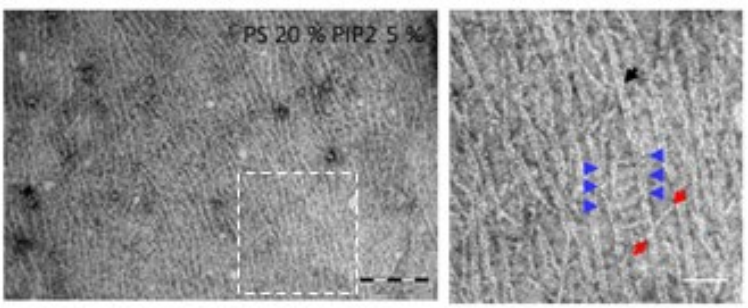

(D)

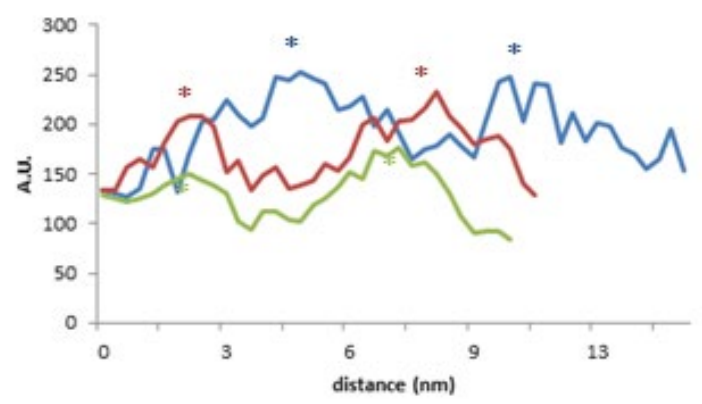

Figure 3. Septin hexamers form dense arrays of tightly paired filaments on anionic lipid monolayers. Electron micrographs of negatively stained fly septin hexamers (65 nM) after overnight incubation with lipid monolayers composed of PC combined with anionic lipids: (A) $20 \%$ PS, (B) $5 \%$ PI(4,5) $P_{2}$, and (C) $20 \%$ $\mathrm{PS}$ plus $5 \% \mathrm{PI}(4,5) \mathrm{P}_{2}$. Images on the right show zoom-ins of the regions in the white-dashed boxes on the left. Black arrows point out examples of paired filaments recognizable by two linear filaments running in parallel. Blue arrows indicate examples of orthogonal hexamers, recognizable by their $\sim 24 \mathrm{~nm}$ length, between adjacent paired filaments. The two red arrows point to the two ends of a longer $(43 \mathrm{~nm})$ crossbridging filament that bridges two adjacent paired filaments under an oblique angle. Scale bars: $250 \mathrm{~nm}$ (left) and $50 \mathrm{~nm}$ (right). (D) Three examples of intensity profiles across paired filaments on monolayers containing $20 \% \mathrm{PS}$ and $5 \% \mathrm{PI}(4,5) \mathrm{P}_{2} \mathrm{PS}$, showing an interfilament spacing (between stars in matching colours) of approximately $6 \mathrm{~nm}$.

The following figure supplement is available for figure 3 :

Figure 3 - Figure supplement 1. EM images of mammalian septin hexamers on lipid monolayers. 

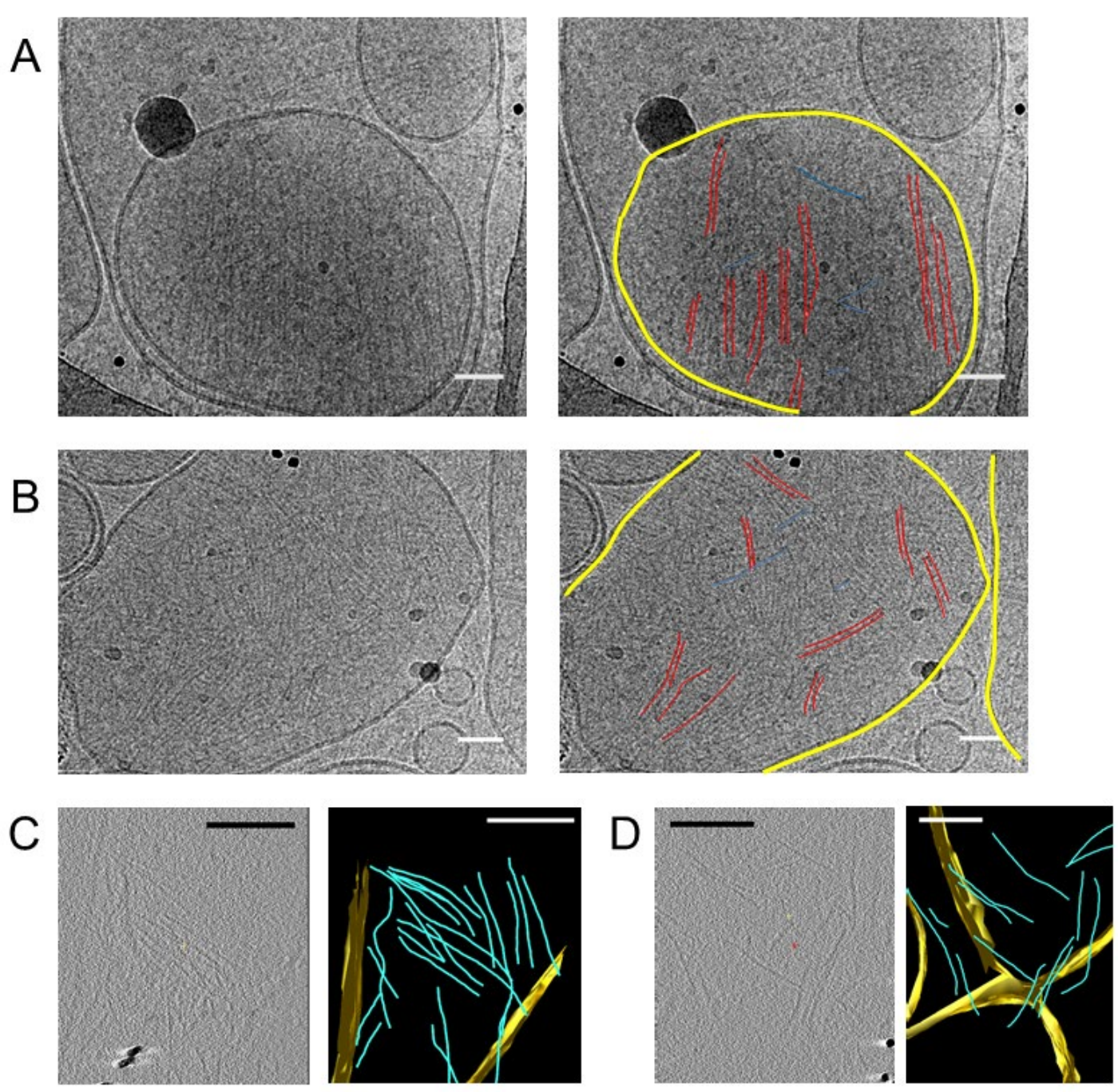

Figure 4. Septin hexamers form single and paired filaments on large unilamellar lipid vesicles. CryoEM images of fly septin hexamers $(300 \mathrm{nM})$ after a 30 minute incubation with PC vesicles containing $6 \%$ $\mathrm{PI}(4,5) \mathrm{P}_{2}$. (A) Example image (left) with paired septin filaments traced out in red, connecting orthogonal filaments in blue and the vesicle membrane highlighted in yellow (right). (B) Another example image (left), with mostly paired and occasionally single filaments traced out in red, connecting orthogonal filaments in blue and membranes in yellow (right). Black dots are gold nanoparticles that were included as fiducial markers for tomography. The black curved lines in panels A, B come from the carbon lacey substrate. (C) Slice from 3D reconstructed cryo-tomogram (left) with segmented data (right). (D) Another example tomogram (left) with segmented data (right). Reconstructions show membrane in yellow and septin filaments in blue. Note that the bilayer perpendicular to the electron beam is poorly defined because of the missing wedge. Scale bars are $50 \mathrm{~nm}$ in $A, B$ and $850 \mathrm{~nm}$ in C, D.

The following video and figure supplements are available for figure 4:

Figure 4 - Figure supplement 1 . Additional cryoEM data.

Figure 4 - Figure supplement 2. Measurements of interfilament spacing for paired fly septin filaments. 
Membrane binding controls ordered self-assembly of animal septins

usually retained their spherical shape in cryoEM. Note that the bilayer perpendicular to the electron beam is poorly defined because of the missing wedge. The deformed membranes provide a first indication that septins were bound to the membrane and able to flatten it. Some septin filaments clearly protrude a bit beyond the vesicle they adhere to, suggesting they were pre-polymerized in solution and attached to one side of the vesicle without fully wrapping around it. Cryo-electron tomography gave further clear evidence that septin filaments are indeed membrane-bound (see Figure $4 C, D$ and Figure 4 - Movie 1).

\section{Septins form thin and mechanically resilient membrane-bound arrays}

We further complemented EM imaging with AFM experiments, which allowed us to image septins on flat lipid bilayers under hydrated conditions resembling the conditions in the TIRF experiments. We performed these experiments on silica-supported lipid bilayers containing $20 \%$ PS, which have already been extensively characterized in their quality by AFM and other biophysical techniques [80]. In particular, the even inter-leaflet distribution of PS lipids in SUVs is approximately preserved upon SLB formation on silica (and glass) supports, whereas this is not the case on mica [81], another commonly used support for AFM. We first tested the dependence of septin assembly on septin concentration by imaging septins at concentrations of 12,24 and $60 \mathrm{nM}$ (Figure 5A-B). The samples were fixed with $1 \%$ glutaraldehyde (GTA) to prevent septin disruption by the AFM tip.

At $12 \mathrm{nM}$, septins formed threads of typically several micrometers in length that were sparsely and randomly distributed on the bilayer surface (Figure $5 A-B$, left column). These were mostly isolated but could also be seen to meet, mostly at a shallow angle, and merge. Their apparent height was mostly uniform at $5.1 \pm 0.9 \mathrm{~nm}(N=29)$, with a few notable exceptions around $12 \mathrm{~nm}(N=2)$. The apparent width showed two relatively broad but distinct populations: most isolated threads had a width of $11.5 \pm 1.6 \mathrm{~nm}$ $(N=19)$, whereas all merged threads and a few isolated ones were wider $(16.6 \pm 0.9 \mathrm{~nm} ; N=12)$ (Figure 5C, left column, and Figure 5 - figure supplement 1 ). These values are consistent with a mixture of mostly single and paired filaments. This can be appreciated if one considers that the minimal filament height is defined by the extension of the globular domain $(4 \mathrm{~nm}$ ) plus some additional contribution by the coiled coils which, owing to the flexible hinge, may point in various directions and additionally become flattened by the force exerted by the AFM tip. Moreover, the flexing of the coiled coils and tip convolution effects (see Methods for details) can explain the relatively broad width distributions, and why the apparent mean widths exceeded the widths of single and paired filaments as seen by EM by about $8 \mathrm{~nm}$ on average.

At $24 \mathrm{nM}$, septins formed threads that were isolated in some places and concentrated in areas of enhanced density in other places (Figure $\mathbf{5 A - B}$, center column). Thread heights and widths were comparable to the $12 \mathrm{nM}$ conditions, although with a higher proportion of higher (up to $13 \mathrm{~nm}$ ) and wider threads (Figure $\mathbf{5 C}$, center column), suggesting the increased presence of paired filaments and the initiation of bundles made from more than two filaments.

At $60 \mathrm{nM}$, the septins formed threads that densely covered the bilayer surface (Figure 5A-B, right column) in closely apposed patches of aligned filaments, resembling nematic domains observed for other $2 \mathrm{D}$ arrays of densely packed semiflexible biopolymers [82]. Salient features of the patches were that the constituent threads varied in height and width, and that they formed a network that is characterized by threads frequently joining and disjoining at a fairly shallow angle of approximately 15 degrees. The smallest thread heights and widths observed were comparable to those observed at $12 \mathrm{nM}$, indicating isolated single or paired filaments were still present. Other threads appeared much wider (up to a few $100 \mathrm{~nm}$; Figure 5C, right column). This suggests that many (up to several tens of) single filaments may 

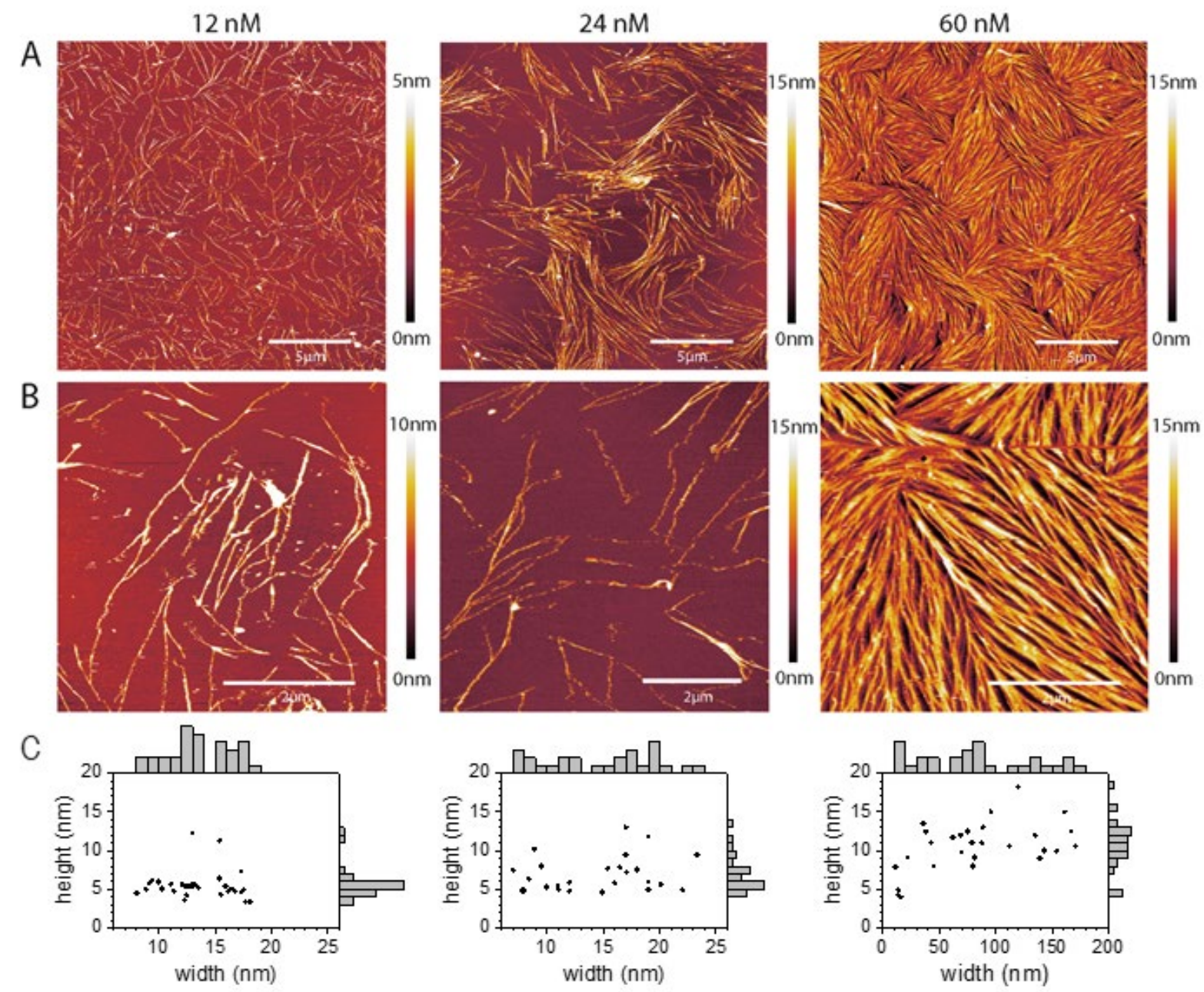

352
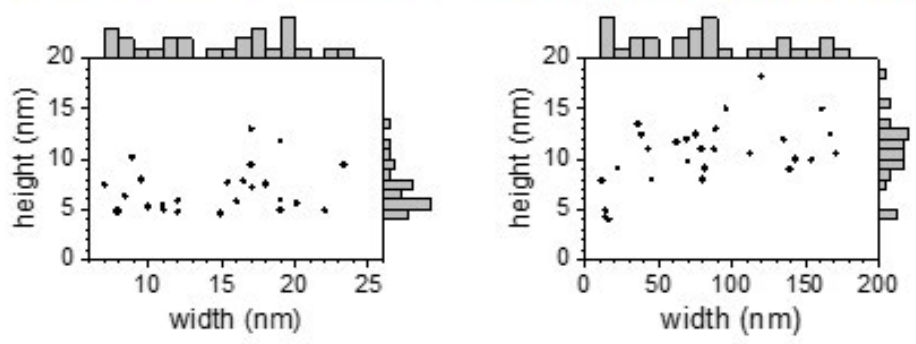

Figure 5. Septins form filaments and ordered arrays on lipid bilayers composed of $\mathbf{8 0 \%}$ PC and $\mathbf{2 0 \%}$ PS. (A-B) AFM topographic images of membrane-bound fly septin structures formed at solution concentrations of $12 \mathrm{nM}$ (left), $24 \mathrm{nM}$ (middle) and $60 \mathrm{nM}$ (right) and observed at a scan size of (A) $20 \times$ $20 \mu \mathrm{m}^{2}$. (B) Same samples, imaged at a scan size of $5 \times 5 \mu \mathrm{m}^{2}$ (left and right) and of $6.7 \times 6.7 \mu \mathrm{m}^{2}$ (middle). Color bars on the right show the height scale. The samples were fixed with glutaraldehyde (GTA). (C) Scatter plots with marginal histograms of thread widths and heights determined from the corresponding images in (B). $N=31$ (left), 26 (middle) and 27 (right) measurements were taken, respectively, across representative sets of well resolved threads.

The following figure supplements are available for figure 5:

Figure 5 - Figure supplement 1. Examples of AFM height profiles across filaments and bundles.

Figure 5 - Figure supplement 2. Images of non-fixed septin samples on lipid bilayers.

Figure 5 - Figure supplement 3. Experiment testing the mechanical stability of the septin structures.

Figure 5 - Figure supplement 4. Experiment testing the adhesion of septin filaments to the membrane. 


\section{Membrane binding controls ordered self-assembly of animal septins}

closely align on the lipid membrane, although the lateral resolution was insufficient to reveal the individual filaments and their spacing. The wider threads also had an elevated height, mostly between 8 and $13 \mathrm{~nm}$ and occasionally up to $18 \mathrm{~nm}$ (Figure 5C, right column), suggesting that septins also stacked on top of each other.

Importantly, the morphologies of unfixed septins resembled those of GTA-fixed ones, though imaging in this case was challenging because the filaments were more easily disrupted by the AFM tip (Figure 5 - figure supplement 2). For one $60 \mathrm{nM}$ unfixed sample, we came across bilayer areas where the septin coverage was low enough to reveal septin filaments that were isolated or ran close together and in parallel with others (Figure 6). Here we observed that the isolated filaments had heights of $4 \mathrm{~nm}$, corresponding to the height of a single septin hexamer and thus a single layer of septin filaments, while the bundled areas had heights between $8 \mathrm{~nm}$ and $12 \mathrm{~nm}$, suggesting that septin filaments can stack on top of each other. We note that in the EM data there is no such clear evidence of layering. However, we note several experimental differences between AFM and EM. In AFM we used solid-supported lipid bilayers as a membrane substrate for septins, whereas in EM we used either lipid monolayers at the airwater interface (for negative stain images) or vesicles (for cryoEM), where the curvature likely prevents septins from reaching a high density of filaments.

Since AFM imaging involves mechanical scanning across the surface, it allowed us to qualitatively test how firmly the septins are attached to the lipid bilayer by performing multiple consecutive scans. In sparsely covered bilayer regions, wider bundles generally remained stable whereas narrower bundles or isolated filaments (both GTA-fixed and unfixed) sometimes appeared ragged, suggesting that the AFM tip moved them along the membrane (Figure 5 -figure supplement $3 \boldsymbol{A}, \boldsymbol{B}$ ). Densely covered regions formed at $60 \mathrm{nM}$ septins were completely unchanged after three consecutive 10-minute scans (Figure 5 - figure supplement $3 \mathbf{C}$ ). These observations suggest that lateral associations among septin filaments within

A
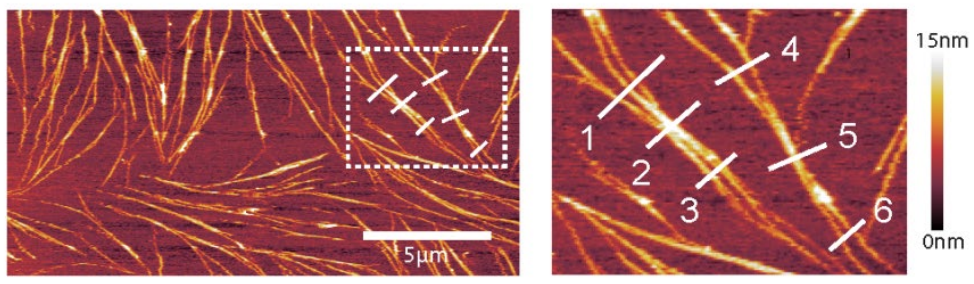

B
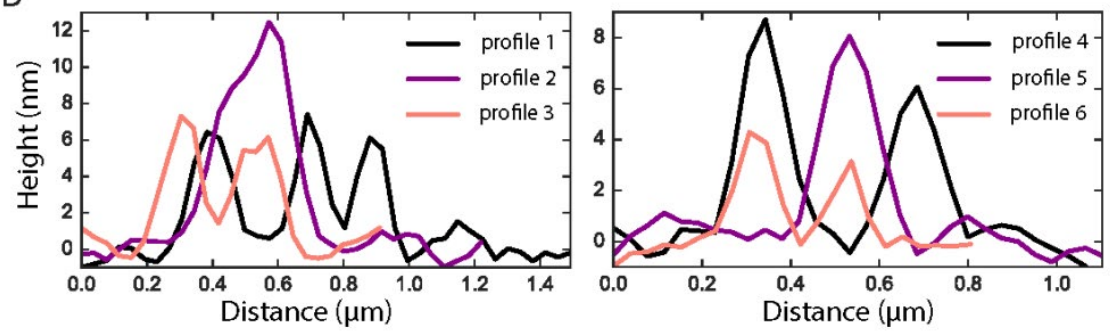

Figure 6. Native ultrastructure of septin assemblies on lipid bilayers composed of $\mathbf{8 0 \%}$ PC and $\mathbf{2 0 \%}$ PS. (A) AFM image of non-fixed septins at $60 \mathrm{nM}$, showing a bilayer region that happens to be sparsely covered with septin filaments. Scale bar for the left panel: $5 \mu \mathrm{m}$; right panel shows zoom of the dashed rectangle in the left panel; color bar on the right shows the height scale. (B) Height profiles were determined along the numbered white lines in the images in $A$. 
Membrane binding controls ordered self-assembly of animal septins

bundles or dense arrays cause mechanical stabilization. Some image sequences clearly showed that entire bundles can be laterally displaced along the membrane owing to lateral forces exerted by the AFM tip. Interestingly, these events did not destroy the bundles but resulted in permanent local kinks, suggesting the septin filaments are intrinsically stiff and ductile but the link to the membrane is fluid (Figure 5-figure supplement 4).

\section{QCM-D measurements show self-limiting septin assembly away from the membrane plane}

The AFM data suggest that fly septins form organized layers of stiff filaments on PS-containing bilayers, with a limited thickness. The maximum thickness of the septin films at $60 \mathrm{nM}$ is approximately $18 \mathrm{~nm}$, according to the height profiles. However, we note that this value may underestimate the actual geometrical height because the tip can potentially indent or otherwise disturb the septin layer. To independently measure the film thickness, and to gain insight into the kinetics of septin film formation, we therefore turned to quartz crystal microbalance with dissipation monitoring (QCM-D), an acoustic technique that can monitor the binding of biomolecules and variations in the mechanical properties of interfacial films in real time [83]. We first formed an SLB on the silica-coated QCM-D sensor by perfusing the channel with a vesicle (SUV) suspension and next perfused with a solution of septin hexamers. To investigate the kinetics of SLB formation and septin binding, we monitored the resulting shifts in the resonance frequency $\Delta f$, which is proportional to the areal mass density of adsorbed biomolecules plus hydrodynamically coupled solvent, and in the dissipation $\Delta D$, an indicator of the mechanical properties of the adsorbed layer.

Typical QCM-D data are presented in Figure 7. The SUVs are composed of $80 \%$ PC and $20 \%$ PS, as in the AFM experiments. SUV perfusion on a plain silica surface (Figure $7 A$ ) caused immediate changes in $\Delta f$ and $\Delta D$ in a two-stage process that is characteristic for the initial adsorption of intact SUVs to the sensor surface followed by SUV rupture, spreading and coalescence [84]. The final shifts of $\Delta f=-25 \pm 1 \mathrm{~Hz}$ and $\Delta D<0.2 \times 10^{-6}(N=6)$ are typical for confluent SLBs composed of PC and PS of a high quality, i.e., with a negligible quantity of residual intact vesicles [80]. Subsequent rinsing with vesicle buffer did not result in appreciable changes in $\Delta f$ and $\Delta D$ confirming the SLB was stable. Septin perfusion at $60 \mathrm{nM}$ on such a SLB (Figure $7 B$ ) produced an immediate decrease of $\Delta f$ and a concurrent increase of $\Delta D$, indicating that septins adsorbed. Binding reached a plateau after about 50 minutes, and subsequent perfusion with buffer caused no appreciable change in $\Delta f$, suggesting that septins stably adsorb to the membrane. In contrast, we did not observe any adhesion of septins to pure PC membranes (Figure $\mathbf{7 B}$; dotted blue line with diamonds). We conclude that septins strongly bind to lipid bilayers containing $20 \%$ PS, and that the presence of negatively charged lipids is required for membrane binding, consistent with the TIRF observations.

The total frequency shift for septin binding was $\Delta f=93 \pm 4 \mathrm{~Hz}(N=4)$. Using the Sauerbrey equation (see Eq. [1]), we estimate a film thickness of $15 \mathrm{~nm}$ from the frequency shift. This value is consistent with the upper end of bundle heights measured by AFM (Figure 5C, right column), which we had attributed to stacking into more than one layer. Moreover, the QCM-D frequency trace also shows that a plateau is reached upon prolonged perfusion with septins. This indicates the presence of a mechanism that limits septin binding and film thickness growth.

Finally, QCM-D data are consistent with the septin assembly having soft molecular linkers within the structure. To a first approximation, the $\Delta D /-\Delta f$ ratio is a measure of elastic compliance (i.e., softness) [83], and for globular proteins the major source of compliance typically are flexible hinges linking the 
Membrane binding controls ordered self-assembly of animal septins

proteins to the surface or inter-connecting protein domains $[83,85]$. Membrane-bound septins attained dissipation over frequency shift $(\Delta D /-\Delta f)$ ratios between $(0.20 \pm 0.01) \times 10^{-6} / \mathrm{Hz}$ at low coverage $(\Delta f=-15$ $\pm 5 \mathrm{~Hz})$ and $(0.09 \pm 0.01) \times 10^{-6} / \mathrm{Hz}$ at high coverage $(\Delta f<-90 \mathrm{~Hz} ; N=4$; Figure 7 -figure supplement 1$)$. For comparison, monolayers of streptavidin attain $\Delta D /-\Delta f$ ratios of $<0.01 \times 10^{-6} / \mathrm{Hz}$ when physisorbed on gold, and between $0.08 \times 10^{-6} / \mathrm{Hz}$ (at low coverage) and $0.015 \times 10^{-6} / \mathrm{Hz}$ (at high coverage) when linked via biotins on a short, flexible linker to a supported lipid bilayer [85]. The higher $\Delta D /-\Delta f$ ratios for septins are consistent with a high degree of flexibility in the linkage between the coiled coils and the globular domains of septin hexamers. Indeed, $\Delta D /-\Delta f$ ratios close to $0.1 \times 10^{-6} / \mathrm{Hz}$ have been reported for monolayers of neutravidin with short (i.e. several ten basepairs long) double-stranded DNA strands grafted to it [86], and for monolayers of streptavidin with linear oligosaccharide chains grafted to it [87]. Possibly the compliance of the coiled coils themselves, and/or the linkage between septins and the membrane, also contribute to the elevated $\Delta D /-\Delta f$ ratio relative to fully globular proteins.

A
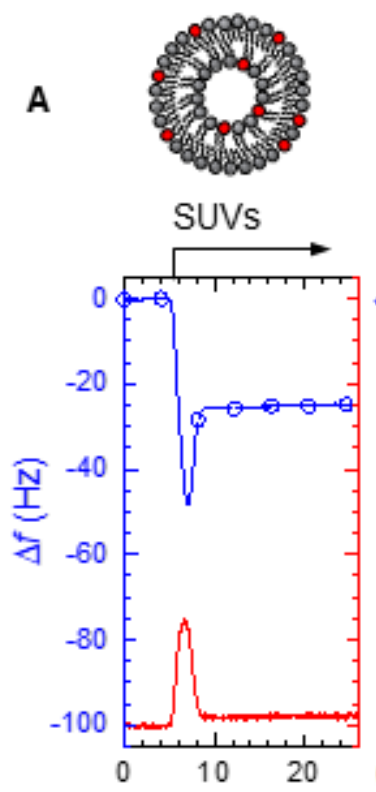

B

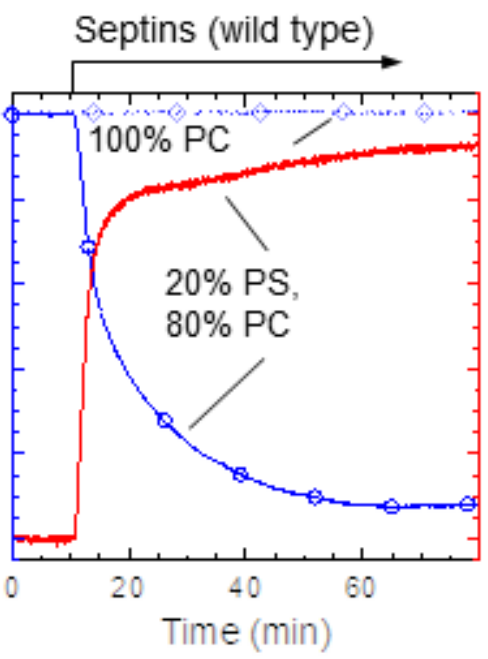

C

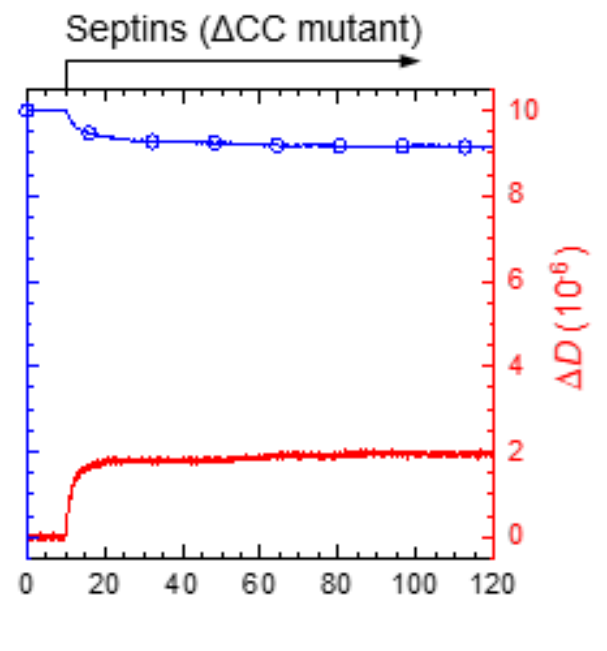

Figure 7. QCM-D measurements of fly septin hexamer adsorption on supported lipid bilayers (SLBs) containing anionic lipids show that septins form thin, rigid films. Shown are frequency shifts ( $\Delta f-$ blue lines with symbols) and dissipation shifts ( $\Delta D$ - red solid lines). Arrows on top of the graphs indicate start and duration of sample perfusion; during remaining times, plain buffer ( $A$ - vesicle buffer; $B$ and $C$ - septin polymerization buffer) was flown over the sensor surface. (A) Small unilamellar vesicles (SUVs at 50 $\mu \mathrm{g} / \mathrm{mL} ; 20 \%$ DOPS, $80 \%$ DOPC) were exposed to a plain silica surface to form a SLB. (B-C) Hexamers of wild type septin (B) and the $\triangle C C$ septin truncation mutant (C) (60 nM each), were exposed to $80 \% \mathrm{PC} / 20 \% \mathrm{PS}$ SLBs (solid line with circle symbols). (B) also shows control frequency data for exposure of wild type septin (100 nM) to pure DOPC SLBs (dotted line with diamond symbols).

The following figure supplement is available for figure 7:

Figure 7 - Figure supplement 1 . Coverage-dependent $\Delta D /-\Delta f$ ratios for fly septins. 
Membrane binding controls ordered self-assembly of animal septins

\section{Septin's coiled coil domains are essential for forming multilayers}

The data described above collectively demonstrate that fly septins form filaments that interact with each other both in the plane of the membrane, and out-of-plane. Which septin domains mediate these interactions? One candidate are the C-terminal coiled coils. In prior work on yeast septins, coiled coils have already been proposed to be involved in septin filament pairing and, on membranes, in the formation of perpendicular octamer cross-bridges between two paired filaments $[9,57]$. A second candidate are the G-domains, which have been proposed to be involved in lateral interactions among yeast septin filaments on membranes [57].

In order to test the arrangement of septins in the presence of G-domain interactions alone, we generated coiled-coil truncated fly septin hexamers ( $\triangle C C$ mutant). Transmission EM confirmed that fly septins with their C-termini truncated from all subunits form stable hexamers (Figure 1 - figure supplement $1 B$ ), similarly to C-terminally truncated human septin hexamers [69]. TEM imaging showed that in solution, the $\triangle C C$ mutant only formed short rods with lengths of $\sim 24-60 \mathrm{~nm}$, corresponding to monomers, dimers or trimers of hexamers, in the low salt polymerization buffer (Figure $\mathbf{8} \boldsymbol{A}, \boldsymbol{B}$ ). However, C-terminally truncated fly septin hexamers on lipid monolayers formed dense arrays of aligned filaments (Figure $\mathbf{8 C}-\boldsymbol{F}$ ). Thus, membrane-binding promotes filament formation, mirroring earlier observations for yeast septin octamers with C-terminally truncated Cdc11 subunits [57]. In places we can see three filaments side by side instead of two (indicated by triple blue lines in Figure $8 D$ ). The mutant filaments were even more closely spaced than wild type septin filaments, with a center-to-center distance of around $4.5 \mathrm{~nm}$ (Figure 8G), consistent with direct contact between the globular domains deprived of the CC domain of adjacent filaments due to crowding and perhaps specific G-domain interactions.

The septin density on the lipid monolayers observed in EM images for the $\Delta C C$ mutant was notably higher than for the full-length septins, even though the solution concentrations were the same. This observation suggests that the $\triangle C C$ mutant perhaps has a reduced tendency to form multilayers. To test this idea, we performed QCM-D measurements for the $\triangle C C$ mutant on bilayers containing $20 \%$ PS. As shown in Figure $7 C$, the $\triangle C C$ mutant binds to the bilayers but the frequency shift is much smaller than for the full-length septins, indicating a thinner layer. In this case, the $-\Delta f$ values remained less than $10 \mathrm{~Hz}$, consistent with a single and sparse septin monolayer. Thus, the EM and QCM-D data demonstrate that the coiled coils are not needed for membrane binding, but they are needed for filament pairing, the formation of cross-bridges, and the stacking of septins on top of each other.

\section{Discussion}

\section{Species-dependence of septin-membrane interactions}

We investigated the influence of membrane-binding on septin assembly by reconstituting recombinant animal septins on supported lipid bilayers and imaging septin assembly with several complementary techniques. Fluorescence imaging revealed that fly septin hexamers have a high affinity for negatively charged lipid bilayers, which competes with the septin -septin lateral interactions that prevail in bulk solution, and form dense membrane-associated meshworks. Electron microscopy (EM) imaging revealed that these meshworks are predominantly comprised of paired filaments and meshworks thereof. A similar organization was observed for mammalian septins, consistent with earlier findings for septin-containing porcine brain extracts $[38,40]$. Finally, atomic force microscopy (AFM) of septins on lipid bilayers showed 
A

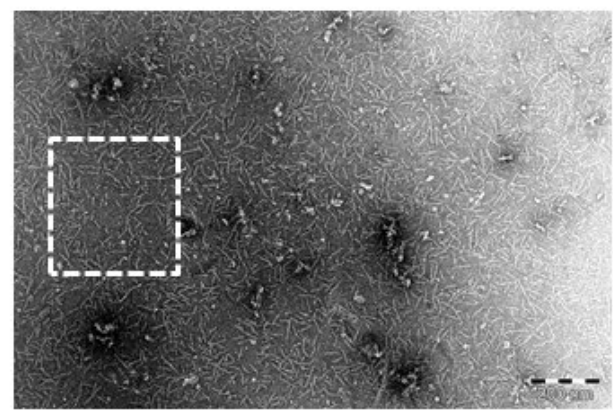

C

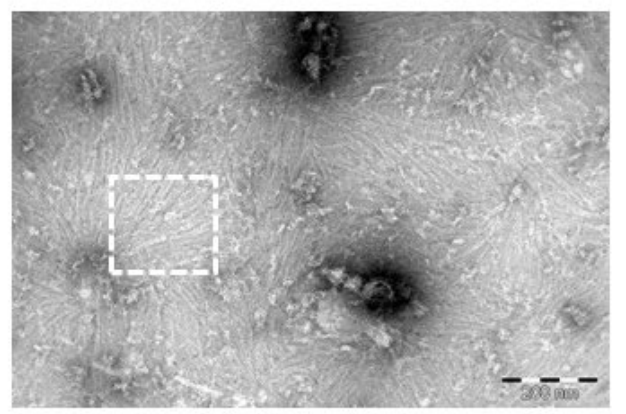

E

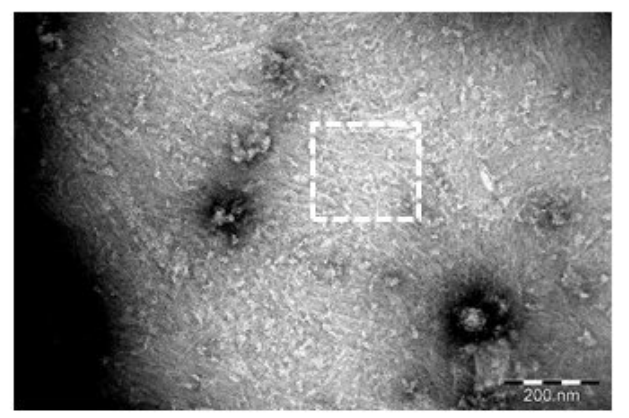

B

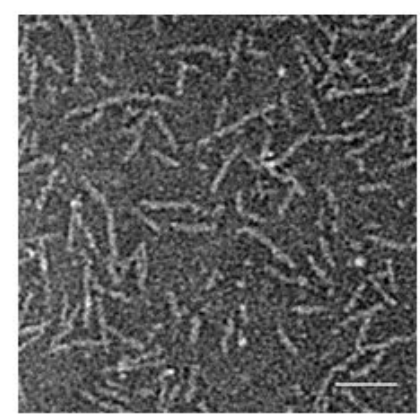

D

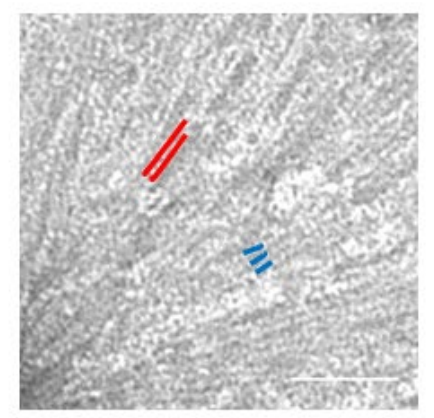

F

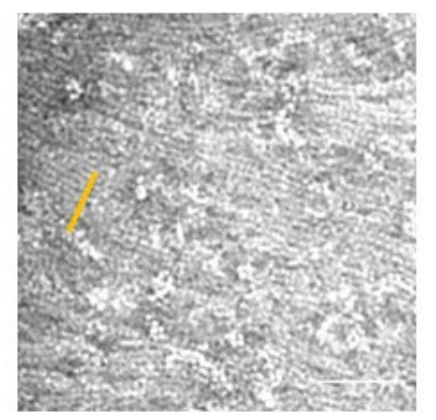

G

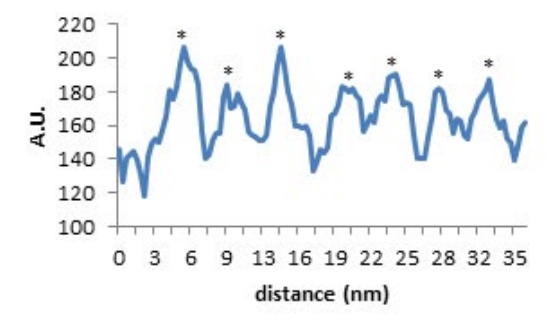

Figure 8. Negative stain EM images of C-terminally truncated fly $\triangle C C$-septins. (A) $\triangle C C$-septin hexamers in septin polymerization buffer. (B) Zoom-in of the boxed region in (A) shows that $\triangle C C$-septin hexamers predominantly form monomers and short oligomers of hexamers with a length of $\sim 24-60 \mathrm{~nm}$. (C) $\Delta C \mathrm{CC}$ septin hexamers (210 $\mathrm{nM}$ ) on a negatively charged lipid monolayer composed of $80 \%$ DOPC, $10 \%$ PS and $10 \% \mathrm{PIP}(4,5) \mathrm{P}_{2}$. (D) Zoom-in of the boxed region in (C) shows sheets of closely spaced, long filaments (red lines) and occasionally three filaments lined up side by side (trimer highlighted in blue). (E) Another example of the $\triangle C C$-septin hexamers $(210 \mathrm{nM})$ on a lipid monolayer composed of $80 \% \mathrm{PC}, 10 \% \mathrm{PS}$ and $10 \% \mathrm{PIP}(4,5) \mathrm{P}_{2}$. (F) Zoom-in of the boxed region in (E) again shows sheets of closely spaced, long, unpaired

517 filaments. (G) Line scan (from area highlighted in panel $F$ by an orange line) reveals a center-to-center 518 (asterisks) spacing of $\sim 4.5 \mathrm{~nm}$. Scale bars are $200 \mathrm{~nm}(A, C, E)$ and $50 \mathrm{~nm}(B, D, F)$. 
Membrane binding controls ordered self-assembly of animal septins

that septin filaments laterally associate into bundles but also stack on top of each other. AFM imaging, fluorescence recovery after photobleaching and QCM-D experiments indicate that the septins are immobile and firmly attached to the membrane. By contrast, C-terminally truncated septin complexes form monolayers and the filaments do not form pairs and meshworks, showing that the coiled coils of septins are crucial for mediating septin-septin interactions.

Prior in vitro studies of septin-membrane binding focused mainly on mitotic, Cdc11-capped budding yeast septin octamers $[57,88]$. We find several striking similarities between the membrane interactions of animal (fly and mammalian) septin hexamers and Cdc11-capped yeast septin octamers. First, membrane binding in both cases promotes septin polymerization. For fly septin hexamers, the threshold for polymerization is lowered more than 10-fold, from $200 \mathrm{nM}$ in solution to less than $12 \mathrm{nM}$ on negatively charged membranes (in the presence of $20 \%$ PS). For yeast septins, a similar enhancing effect was observed on membranes containing $\mathrm{PI}$ or $\mathrm{PI}(4,5) \mathrm{P}_{2}[57,88]$. The polymerization-enhancing effect of membrane binding is likely due to the increase of the effective septin concentration caused by $2 \mathrm{D}$ confinement. In addition, membrane binding may orient septins in a manner that promotes their polymerization, as seen for other proteins such as annexin A5 [89]. A second similarity between the behavior of animal and yeast septins on membranes is that they both form paired filaments visible by transmission electron microscopy, with a narrow spacing of less than $2 \mathrm{~nm}$, much narrower than the 10 $\mathrm{nm}$ spacing observed for yeast septin filaments formed in solution [9]. A third similarity is that paired filaments of both animal and yeast septins are interconnected by bridges visible by electron microscopy, which appear to be formed by short and thin (single) septin filaments having a length and axial spacing that correspond to the length of a single protomer [57]. Similar arrays have been observed by electron microscopy in yeast cells [90], but not yet in animal cells. Note that yeast septins were studied only by transmission electron microscopy, which provides a 2D-projection. It is unknown whether yeast septins form multilayers on membranes, as we see for fly septins by AFM and QCM-D.

Our work also reveals two striking differences between animal and yeast septins. The first difference is their polymerization in solution. Fly septins form thick and rigid bundles with tapered ends in solution, suggesting that the septins have strong lateral interactions that promote bundling. On membranes, however, we observed by TIRF microscopy a gradual transition from dilute arrays of thick bundles to dense arrays of paired filaments with increasing net surface charge. This observation suggests a competition of septin-membrane interactions with lateral septin-septin interactions, which suppresses the formation of thick bundles on membranes. By contrast, yeast septin octamers tend to form paired filaments in solution [9,57], although they do form bundles under certain conditions [91]. Thus, in solution fly septins have a stronger propensity for bundling than yeast septins, but on membranes the two septins behave similarly. The second striking difference between yeast and animal septins revealed here is their membrane binding selectivity. We find that fly septins form similar structures on membranes containing $\mathrm{PS}$ or $\mathrm{PI}(4,5) \mathrm{P}_{2}$, and that the main determinant of fly septin binding and filament organization in both cases is the net surface charge of the membrane. By contrast, Cdc11-capped yeast septin octamers were shown to be highly selective for $\mathrm{PI}, \mathrm{PI}(4) \mathrm{P}, \mathrm{PI}(5) \mathrm{P}$ and $\mathrm{PI}(4,5) \mathrm{P}_{2}$, while they did not interact with $\mathrm{PS}[10,39,57,58$, $79,88,92,93]$. The origin of the differences in lipid specificity remains unknown. It is thought that septins interact with negatively charged phospholipids via a polybasic region close to the $\mathrm{N}$-terminus that is composed of a sequence of 1 to 7 basic amino acids [37, 92]. This stretch is very similar to polybasic sequences found in gelsolin, profilin, G-protein-coupled receptor kinases and ion channels [37], which are 
Membrane binding controls ordered self-assembly of animal septins

all reported to interact with $\mathrm{PI}(4,5) \mathrm{P}_{2}$. In the future perhaps molecular dynamics simulations can identify the determinants of lipid selectivity [94].

\section{Towards a model of septin assembly on membranes}

What do the imaging experiments collectively teach us about the assembly mechanism of fly septin hexamers on lipid membranes? A firm conclusion is difficult because there are still a number of important unknowns. It is still under debate where the membrane-binding region is located $[79,95,96]$. We observe that fly septin hexamers are recruited to the membrane by electrostatic interactions with anionic lipids (PS and/or $\mathrm{PI}(4,5) \mathrm{P}_{2}$ ). It is generally believed that the $\mathrm{N}$-terminal polybasic domains of septins interact with the membrane, which would leave the coiled coils free to rotate in the half-space above the membrane, although a recent study on yeast septin octamers suggested that membrane binding is aided by an amphipathic helix motif identified on the C-terminal side of the coiled-coil of Cdc12 [79]. In our case, however, we observe that C-terminally truncated septins still bind membranes and our data on full-length septins are consistent with the coiled coils being available for interactions with coiled coils on neighboring septins. Since the coiled coils are connected to the body of the septin complex via unstructured regions that can act as hinges, we assume that the coiled coils can rotate in the entire half-plane above the membrane, so they are available for both in-plane and out-of-plane interactions with coiled coils on neighboring septin subunits/filaments. The nature of these interactions is still unknown (i.e., assembly of two vs. four coiled coils, and parallel vs. antiparallel assembly). Nevertheless, our imaging and QCM-D experiments do allow us to present a speculative model of membrane-templated septin self-assembly, which is illustrated in Figure 9.

On the left in Figure 9A, we display the proposed organization of septin filaments in the membrane-proximal layer. The hexamers form paired filaments by a combination of end-to-end association mediated by homodimerization of the terminal DSep1 subunits of adjacent hexamers, and lateral associations. Paired filaments in turn form in-plane bundles that occasionally branch and merge. In addition, paired septin filaments are bridged by hexamers or dimers thereof. Similar bridges were observed for yeast septin octamers on lipid monolayers, where the bridge length and spacing were also multiples of the octamer length [57]. In the context of yeast septins, it was proposed that coiled coils mediate septin filament pairing and the formation of perpendicular octamer cross-bridges between paired filaments $[9,57]$. The interpretation in that case was that at least some C-terminal extensions on the terminal Cdc11 subunits of paired filaments were free to engage in interactions with C-terminal extensions on the bridging filaments. We here propose that fly septins may similarly form bridges via interactions of the C-terminal coiled coils of the terminal subunits (DSep1), facilitated by their orientational flexibility conferred by the hinge regions. Indeed for the C-terminally truncated septin hexamers, both pairing and cross-bridging are abrogated.

To explain why septins form multi-layers, we hypothesize that not all coiled coils are engaged in interactions within the first membrane-proximal septin layer, thus leaving some coiled coils free to engage off plane. Pairing of the septin filaments in the membrane plane is very tight, with a spacing of only $2 \mathrm{~nm}$, much smaller than the 13-nm extended length of the long coiled coils on Pnut and DSep2 subunits. Similarly tight pairing was previously observed for yeast septins on lipid monolayers [57]. To explain this, we consider that the coiled-coils may be oriented either upwards and engage transversely with coiled coils on a second layer of septins (Figure 8 right, scenario 1), or parallel to the membrane sandwiched 
(A) full-length septins

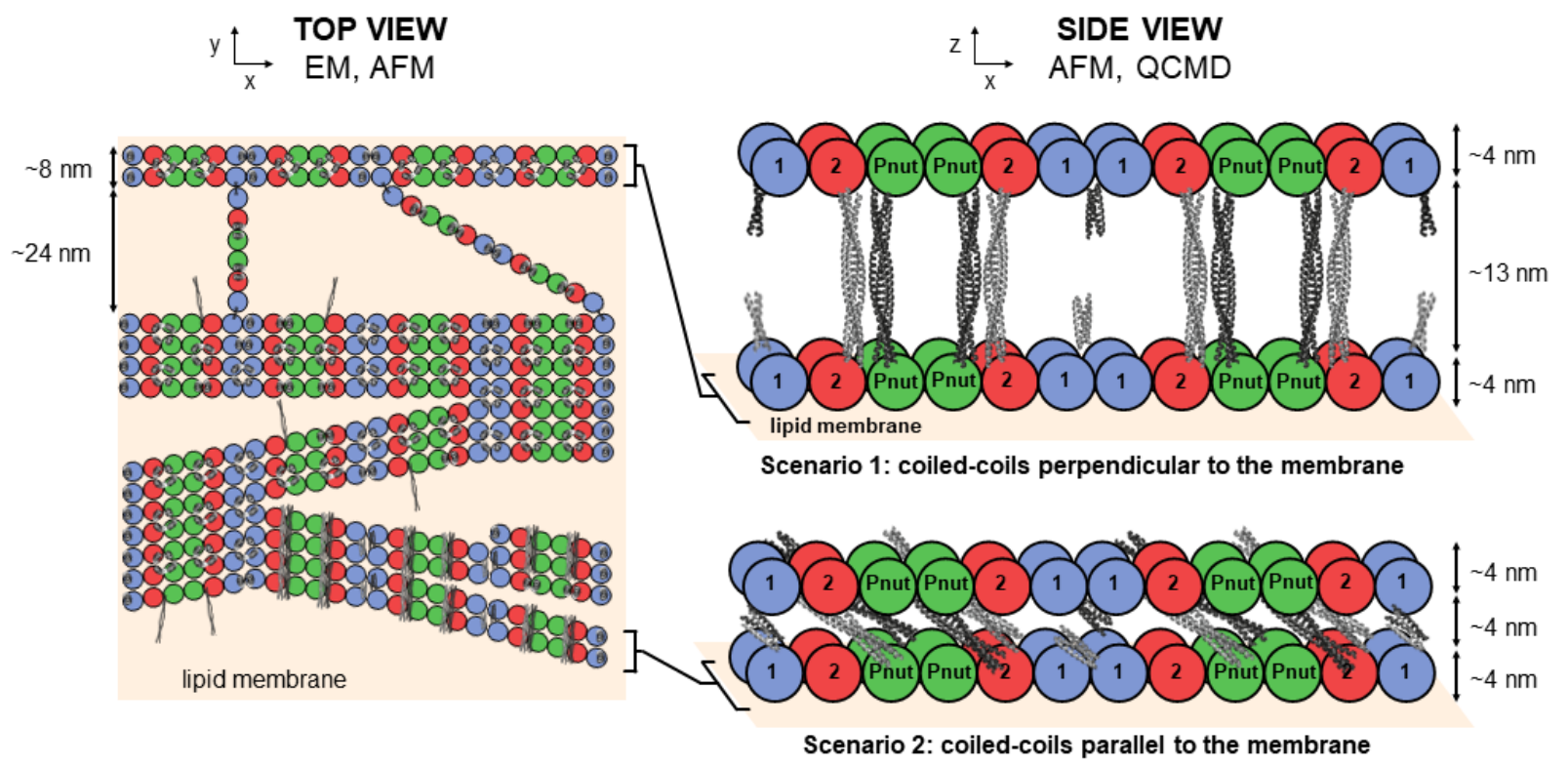

(B) $\triangle$ CC septins
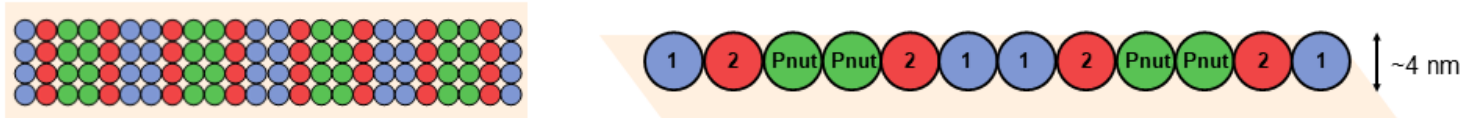

604

605

606

607

608

609

610

611

612

613

614

615

616

617

618

619

620

621

622

623

624

Figure 9. Model of membrane-templated septin self-assembly. Left column: top views of the membrane proximal septin layer. Right column: side views of all septin layers. The coiled-coils are depicted to scale using available crystal-structures of coiled-coils (see Methods) in light-gray for coiled coils emanating from the bottom septin layer and dark-gray for coiled-coils from the top layer. (A) Full-length septin hexamers form meshworks of paired filaments on the membrane, which are bridged by hexamers and dimers thereof and form bundles with filaments joining or emerging at a shallow angle. A second layer of septin filaments is recruited by coiled-coil interactions oriented vertically (scenario 1) or in-plane (scenario 2 ). For simplicity, scenario 2 is depicted with coiled coils oriented perpendicular to the filament axis though they may in reality adopt a range of orientations. (B) $\Delta C C$-septin hexamers lacking coiled coils form a monolayer of filaments interacting by excluded volume interactions, perhaps augmented by G-domain interactions. Dimensions indicated in the schematic are based on the known size of septin subunits from EM and calculated lengths of the coiled-coil regions, and are consistent with the measured filament widths and spacings in-plane (from EM images) and with the measured height of the septin films (from AFM and QCM-D).

between two layers of septins (Figure 8 right, scenario 2). According to scenario 1, we would expect a layer height of $21 \mathrm{~nm}$, which is larger than the maximum height of $\sim 18 \mathrm{~nm}$ seen by AFM for dense yet subsaturated membranes, and the average height of $\sim 15 \mathrm{~nm}$ seen by QCM-D for saturated membranes. According to scenario 2, we would expect a layer height of $\sim 12 \mathrm{~nm}$, in better agreement with the typical 
Membrane binding controls ordered self-assembly of animal septins

heights seen in AFM. However, the AFM images show that the bundle heights are variable. We therefore anticipate a combination of both scenarios, or even alternate scenarios, given the length and orientational freedom of the coiled coils and the known ability of coiled coils to form both parallel and antiparallel multimers [97, 98]. Importantly, layering is restricted to just two layers. Our interpretation is that coiled coils are mostly engaged in in-plane associations while fewer are engaged in the recruitment of a new layer of septins, so that with increasing layer height, there is a smaller and smaller probability of recruiting additional material on top. This ultimately generates septin arrays that are very extensive in-plane, but limited in thickness to a 12 nm thick array with a layer of coiled coils sandwiched between two layers of globular domains. Thicker layers appear to be rare, and may arise either from a different organization of the second septin layer or from an additional septin layer.

\section{Biological implications}

Our observations of mechanically stable septin filament networks on model biomembranes suggest the possibility that septins may directly enhance the cortical rigidity of cells by forming filaments on the inner leaflet of the plasma membrane. With their high stability, septins could furthermore have an important role in mechanical integration of the cell cortex, since the actin-myosin cortex in mesenchymal and epithelial cells is known to turn over rapidly [99-102]. Although septins in cells are more dynamic than in vitro, with typical in vivo half-times for fluorescence recovery on the order of $100 \mathrm{~s}$ (depending on septin subunit), the turnover rate of septins is still slower than for actin (where the half-time for fluorescence recovery is on the order of tens of seconds) [36]. In the context of amoeboid T-cell migration, it has for instance been suggested that stable septin structures are important to ensure directional extension of leading-edge protrusions [25]. New 3D-superresolution techniques [103] and EM tomography [13] of the cell cortex may be able to resolve the proximity of cortical septins to the cell membrane to answer the question whether septins indeed interact directly with the membrane at the cell cortex.

\section{Materials and Methods}

\section{Septin purification}

Chemicals were bought from Sigma unless indicated otherwise. Recombinant Drosophila septin hexamers composed of DSep1 with an N-terminal His 6 tag, DSep2, and Pnut with a C-terminal Strep tag II (WSHPQFEK, $1058 \mathrm{Da}$ ) were purified from Escherichia coli BL21(DE3) cells (Agilent Technologies) using a two-tag affinity purification scheme to aid the isolation of full-length complexes as explained in ref. [70]. Using the same cloning strategy as for wild type complexes [62], a coiled-coil truncation mutant of fly septin hexamers referred to as the $\triangle C C$ mutant was made with $\mathrm{C}$-terminal truncations right after the end of the $\alpha 6$-helix (DSep1 $\Delta$ C56, DSep2 $\Delta$ C111, Pnut $\Delta$ C123). Cell cultures for wild type and $\Delta C C$ septins were grown at $37^{\circ} \mathrm{C}$ and expression was induced with $0.5 \mathrm{mM}$ isopropyl- $\beta$-D-1-thiogalactopyranoside (IPTG) when the optical density at $600 \mathrm{~nm}$ (OD600) reached a value between 2 and 3. Fluorescently tagged septin complexes were obtained by tagging DSep 2 with monomeric enhanced green fluorescent protein (mEGFP) on its N-terminus [62]. To ensure GFP-folding, cell cultures for GFP-labeled septins were grown at $37^{\circ} \mathrm{C}$ until the OD600 reached 0.6-0.8 and expression was induced overnight at $17^{\circ} \mathrm{C}$.

Cell pellets collected after centrifugation $\left(10 \mathrm{~min}, 2800 \times \mathrm{g}, 4^{\circ} \mathrm{C}\right.$ ) were lysed on ice with a tip sonicator in lysis buffer $\left(50 \mathrm{mM}\right.$ Tris- $\mathrm{HCl} \mathrm{pH} \mathrm{8,} 500 \mathrm{mM} \mathrm{KCl}, 10 \mathrm{mM}$ imidazole, $5 \mathrm{mM} \mathrm{MgCl}, 20 \mathrm{mM} \mathrm{MgSO}_{4}$, $0.1 \mathrm{mM}$ guanosine diphosphate (GDP), $5 \%$ glycerol) supplemented with $0.25 \mathrm{mg} / \mathrm{mL}$ lysozyme (5934-D, 
Membrane binding controls ordered self-assembly of animal septins

Euromedex), $1 \mathrm{mM}$ phenylmethylsulfonyl fluoride (Sigma), Complete ${ }^{\circledR}$ protease inhibitor cocktail (Roche), and $10 \mathrm{mg} / \mathrm{L}$ DNase I (Sigma). The clarified lysate obtained after centrifugation (30 min at $20000 \times \mathrm{g}, 4^{\circ} \mathrm{C}$ ) was first run on a streptavidin affinity column (StrepTrap ${ }^{\circledR} \mathrm{HP}$, GE Healthcare) equilibrated with septin 1 buffer ( $50 \mathrm{mM}$ Tris- $\mathrm{HCl} \mathrm{pH} 8,300 \mathrm{mM} \mathrm{KCl}, 5 \mathrm{mM} \mathrm{MgCl} 2,5 \%$ glycerol) by eluting with $2.5 \mathrm{mM}$ dDesthiobiotin. The peak fractions were pooled and run on a nickel affinity column (HisTrap FF crude column, GE Healthcare) equilibrated with septin 2 buffer $(50 \mathrm{mM}$ Tris- $\mathrm{HCl} \mathrm{pH} \mathrm{8,500} \mathrm{mM} \mathrm{KCl,} 10 \mathrm{mM}$ imidazole, $5 \mathrm{mM} \mathrm{MgCl}_{2}$, $5 \%$ glycerol). Pooled fractions from the elution peak were dialyzed against storage buffer ( $50 \mathrm{mM}$ Tris- $\mathrm{HCl} \mathrm{pH} 8,300 \mathrm{mM} \mathrm{KCl}, 5 \mathrm{mM} \mathrm{MgCl} 2,5 \%$ glycerol, $5 \mathrm{mM}$ dithiothreitol (DTT)) at $4^{\circ} \mathrm{C}$ overnight and concentrated to $\sim 3-5 \mathrm{mg} / \mathrm{mL}$ using Vivaspin 6 concentrators (Sartorius, $6 \mathrm{ml}, 100 \mathrm{kDa}$ cutoff). Mammalian septin hexamers composed of mouse Sept2 (98.6\% identical to human Sept2, differing in 5 out of 361 residues, based on sequence alignment with the Clustal Omega from https://www.ebi.ac.uk/Tools/msa/clustalo/) with an N-terminal His 6 tag, human Sept6, and human Sept7 with a C-terminal Strep tag II were purified in the same manner [41]. Budding yeast septin octamers (composed of $\mathrm{Cdc} 3, \mathrm{Cdc} 10, \mathrm{Cdc11}$ and $\mathrm{His}_{6}-\mathrm{Cdc} 12$ ) were purified as described [9] and stored in $50 \mathrm{mM}$ Tris- $\mathrm{HCl}$ buffer $(\mathrm{pH}$ 8) supplemented with $300 \mathrm{mM} \mathrm{KCl}$. Purified septin complexes were flash-frozen in liquid nitrogen and stored at $-80^{\circ} \mathrm{C}$.

\section{Septin characterization}

The concentration of septin complexes was determined by measuring the optical absorbance of the solutions at $280 \mathrm{~nm}$ with a UV-VIS spectrophotometer (Thermo Scientific, Nanodrop 2000) and subtracting the contribution of scattering (typically 2-3\%) from absorbance measurements between 320-340 nm [104]. We used extinction coefficients of $0.545 \mathrm{~L} \times \mathrm{g}^{-1} \times \mathrm{cm}^{-1}$ for full-length Drosophila septins, 0.584 $\mathrm{L} \times \mathrm{g}^{-1} \times \mathrm{cm}^{-1}$ for mEGFP-tagged septins, $0.645 \mathrm{~L} \times \mathrm{g} \mathrm{g}^{-1} \times \mathrm{cm}^{-1}$ for the $\Delta \mathrm{CC}$ mutant, and $0.565 \mathrm{~L}^{\circ} \mathrm{g}^{-1} \times \mathrm{cm}^{-1}$ for the mammalian septins, with the values all calculated from the sequence [105]. Molar concentrations were converted into weight concentrations using the calculated molecular weights of $306.9 \mathrm{kDa}$ for fulllength untagged Drosophila septins, $361.6 \mathrm{kDa}$ for mEGFP-tagged septins, $237.8 \mathrm{kDa}$ for the $\Delta \mathrm{CC}$ mutant, and $285.7 \mathrm{kDa}$ for the mammalian septins. The purity and integrity of the proteins was evaluated for each batch by sodium dodecyl sulfate polyacrylamide gel electrophoresis (SDS-PAGE) using 4-15\% gradient gels (Mini-PROTEAN ${ }^{\circledR}$ TGX ${ }^{\circledR}$ Precast Protein Gels, Bio-Rad) with a Precision Plus Protein ${ }^{\circledR}$ Kaleidoscope ${ }^{\circledR}$ (BioRad) protein standard (see Figure 1 - figure supplement $1 A, B$ for full-length and $\triangle \mathrm{CC}$ septins, respectively). The length of the purified complexes (as a readout of the stability of the hexameric arrangement) was evaluated by transmission electron microscopy (TEM). Solutions of septins diluted to $65 \mathrm{nM}$ with a buffer containing $300 \mathrm{mM} \mathrm{KCl}$, to prevent polymerization, were deposited on glowdischarged carbon coated copper grids (CF300-Cu, Electron Microscopy Sciences) and incubated for at least $30 \mathrm{~min}$. The samples were negatively stained with $2 \%$ uranyl acetate (Nanoprobes, Yaphank, USA), air-dried, and examined with a FEI Tecnai Spirit (120 kV) electron microscope (ThermoFisher, Waltham, USA). We observed rod-shaped oligomers, mostly having the expected $24 \mathrm{~nm}$ length of a hexamer (Figure 1 -figure supplement $1 A, B$ ). We performed a total of 10 preparations of the full-length fly septins and verified that the protein quality by SDS-PAGE and by TEM analysis was constant. We used 2 preparations of C-terminally truncated fly septins, 1 preparation of yeast septins, and 1 preparation of mammalian septins. 

available under aCC-BY 4.0 International license.

Membrane binding controls ordered self-assembly of animal septins

\section{Sequence alignment of fly and human septins}

Sequence alignment of fly and human septins was performed using the Clustal Omega multiple sequence alignment program (https://www.ebi.ac.uk/Tools/msa/clustalo/) using the full-length sequences of these septins. The \% identity scores mentioned in the text are extracted from the percent identity matrix output of the multiple sequence alignment. A score of $100 \%$ is given to two identical amino acids and a score of zero otherwise.

\section{Septin coiled-coil size prediction}

To identify the regions of the C-termini of fly and human septins that can adopt a coiled-coil conformation, we employed the coiled-coil prediction algorithm COILS (https://embnet.vitalit.ch/software/COILS form.html) using the full-length sequences of these septins. The output was identical when we used the scoring matrix MTIDK (derived from myosins, paramyosins, tropomyosins, intermediate filaments, desmosomal proteins and kinesins) or MTK (derived from the sequences of myosins, tropomyosins and keratins [106]), and for both weighted and unweighted scans, indicating little bias due to the high incidence of charged residues. Figure $1 \mathbf{A}$ displays the predicted structures for fly septins starting right after the $\alpha 6$-helix (ending in ...DRLAK for DSep1; ...RLEQ for DSep2; ...KLSE for Pnut). For DSep1, the algorithm predicted a 28-residue-long coiled-coil (LGEKDR...AQMQAR) with 99.8\% probability. An 86-residue-long C-terminal coiled-coil (QQTFEA..QQLATA) was predicted for DSep2 (47\% probability for the first 21 residues and $86-99.9 \%$ probability for the following 65 ones). An 86-residuelong stretch in the C-terminus of Pnut (LTQMEE...HVTLEE) was further predicted to adopt a coiled-coil conformation with $>97.9 \%$ probability. Figure $1 B$ shows the corresponding predictions for human septins starting right after the $\alpha 6$-helix (ending in ... RLKR for Sept2; ...KLEE for Sept6; ...KLAA for Sept7). The Cterminus of hSept2 was predicted to form a 28-residue-long coiled-coil (LLEKEA...QMQMQG) with 90.2\% probability. The coiled-coil prediction for mSept2 is identical. An 86-residue-long stretch in the C-terminus of Sept6 (QETYEA...TAAELL) was predicted to adopt a coiled-coil conformation with $74-100 \%$ probability. An 86-residue-long C-terminal coiled-coil (LAQMEE..RILEQQ) was further predicted for Sept7 with 99.5$100 \%$ probability. The coiled-coil predictions were fully in line with the $\alpha$-helix prediction output of the secondary structure prediction programs PHYRE2 and PSIPREDV4.0 $[107,108]$. From the end of the a6helix to the start of the predicted coiled-coils, there are stretches of 24, 15 and 15 residues for DSep1, DSep2 and Pnut, respectively, and stretches of 15, 15 and 19 residues for Sept2, Sept6 and Sept7, respectively, that are predicted to be unstructured. To depict septin coiled-coils, the crystal structures of which have not yet been published, in Figure 1C, Figure 1-Figure supplement 1A, Figure 7B and Figure 9, we have used the available crystal structures of coiled-coil dimers and tetramers from vimentin (PDB 3UF1) (coiled-coil side-views in the figures) and the early endosomal SNARE complex (PBD 2NPS) (coiledcoil top-views in the figures). The molecular structure visualization program PyMOL was used for isolating helical segments, and rotating and coloring the dimeric and tetrameric coiled-coil segments shown in the images.

\section{Preparation of small unilamellar lipid vesicles}

Lipids were purchased from Avanti Polar Lipids (Birmingham, AL). The following lipids were bought and stored at a concentration between 10 and $25 \mathrm{mg} / \mathrm{mL}$ in chloroform: 1,2-dioleoyl-sn-glycero-3phosphocholine (PC), 1,2-dioleoyl-sn-glycero-3-phospho-L-serine (PS), and 1,2-dioleoyl-sn-glycero-3phosphoethanolamine- $\mathrm{N}$-(lissamine rhodamine B sulfonyl) (rhodamine-PE). Furthermore, 1,2-dioleoyl-sn- 
Membrane binding controls ordered self-assembly of animal septins

glycero-3-phospho-(1'-myo-inositol-4',5'-bisphosphate) (ammonium salt) $\left(\mathrm{PI}(4,5) \mathrm{P}_{2}\right)$ was bought in powder form and stored as a $0.5 \mathrm{mg} / \mathrm{mL}$ solution in a chloroform:methanol:water mixture (20:9:1 volume ratio). Small unilamellar vesicles (SUVs) were prepared by drying mixtures of PC/PS/rhodamine-DOPE (79.7:20.0:0.3 molar ratio), $\mathrm{PC} / \mathrm{PI}(4,5) \mathrm{P}_{2} /$ rhodamine-PE (94.7:5.0:0.3), or $\mathrm{PC} / \mathrm{PS} / \mathrm{PI}(4,5) \mathrm{P}_{2} /$ rhodamineDOPE (74.7:20.0:5.0:0.3) in glass vials using an air stream. PC/PS lipid mixtures were resuspended in a filtered and degassed imidazole buffer of $\mathrm{pH} 7.4(20 \mathrm{mM}$ imidazole- $\mathrm{HCl}, 50 \mathrm{mM} \mathrm{KCl}$, and $2 \mathrm{mM} \mathrm{MgCl}$ ). The total lipid concentration of the stock solutions was $0.5 \mathrm{mM}$ for TIRF imaging and $2.5 \mathrm{mM}$ for Atomic Force Microscopy (AFM) and Quartz-Crystal Microbalance with Dissipation monitoring (QCM-D) experiments. $\mathrm{PI}(4,5) \mathrm{P}_{2}$-containing lipid mixtures were resuspended at a total lipid concentration of 0.25 $0.5 \mathrm{mM}$ in a $50 \mathrm{mM}$ citrate buffer of $\mathrm{pH} 4.8$ containing equimolar amounts of trisodium citrate and citric acid, $50 \mathrm{mM} \mathrm{KCl}$, and $0.1 \mathrm{mM}$ ethylenediaminetetraacetic acid (EDTA). The acidic $\mathrm{pH}$ promotes the formation of homogeneous and fluid bilayers by reducing the net charge on the head group of $\mathrm{PI}(4,5) \mathrm{P}_{2}$ from -4 to -3 [109]. SUVs for TIRF experiments were obtained by sonication with a tapered microtip (Branson, USA; $3 \mathrm{~mm}$ diameter) for $30 \mathrm{~min}$ in pulsed mode (30 s on/30 s off at 10\% amplitude). SUVs for AFM and QCM-D experiments were obtained by exposing the lipid suspensions to 5 freeze/thaw cycles followed by sonication with a microtip (FisherBrand; $2 \mathrm{~mm}$ diameter) for $30 \mathrm{~min}$ in pulsed mode ( $1 \mathrm{~s}$ on/ 1 $\mathrm{s}$ off at $30 \%$ amplitude) with ice cooling. The SUVs were centrifuged (15 min at $15000 \times \mathrm{g}$ ) to remove any titanium particles that might come off the sonicator tip. All vesicles were stored in the fridge. $\mathrm{PI}(4,5) \mathrm{P}_{2^{-}}$ containing SUVs were used within 7 days, and all other vesicles within 30 days.

\section{Fluorescence microscopy}

Samples were prepared in home-made flow channels assembled from cleaned coverslips and microscope slides that were rendered hydrophilic in base piranha ( $5 \%$ hydrogen peroxide, $5 \%$ ammonium hydroxide in water) at $70^{\circ} \mathrm{C}$. Flow channels were prepared by assembling a dried coverslip and microscope slide with two parafilm spacers spaced apart by $\sim 2 \mathrm{~mm}$ and fixed in place by melting on a hotplate at $120^{\circ} \mathrm{C}$. Septins were fluorescently labeled by co-polymerizing 90 mol-\% untagged hexamers with 10 mol-\% mEGFPtagged hexamers. Septin assembly on supported lipid bilayers (SLB) was performed on the microscope. SLBs were first formed by flushing the SUV stock solution into the flow channels and allowing for SUV rupture and spreading (10 to $15 \mathrm{~min}$ ). Correct SLB formation was ascertained by checking that the fluorescence signal was spatially uniform and, using fluorescence recovery after photobleaching measurements, that the bilayer was fluid. Residual SUVs were removed by washing the SLBs with 5 channel volumes of septin polymerization buffer ( $20 \mathrm{mM}$ imidazole pH 7.4, $1 \mathrm{mM}$ DTT, $0.1 \mathrm{mM}$ MgATP, 50 $\mathrm{mM} \mathrm{KCl}$ and $2 \mathrm{mM} \mathrm{MgCl}$ ), containing $2 \mathrm{mM}$ trolox to suppress blinking [110] and a $2 \mathrm{mM}$ protocatechuic acid/0.1 $\mu \mathrm{M}$ protocatechuate 3,4-dioxygenase mixture to suppress photobleaching [111]. Septin hexamers diluted to a concentration between $10 \mathrm{nM}$ and $1 \mu \mathrm{M}$ in polymerization buffer were then flushed onto the SLBs and assembly was allowed to proceed for 30 minutes at $20^{\circ} \mathrm{C}$. In case of net-neutral (pure PC) bilayers, we had to include 0.1 wt-\% methylcellulose in the buffer to crowd the septins close enough to the surface to allow visualization by TIRF microscopy. In case of anionic bilayers, we did not use methylcellulose.

TIRF imaging was performed with a Nikon Eclipse Ti-E inverted microscope equipped with a TITIRF-E motorized TIRF Illuminator (Roper Scientific), a Nikon Apo TIRF 100×/1.49 N.A. oil immersion objective, and a $491 \mathrm{~nm} / 50 \mathrm{~mW}$ Calypso laser (Cobolt, Solna, Sweden). Images were acquired with a QuantEM 512SC EMCCD camera (Photometrics, Roper Scientific, Tucson, AZ, USA) using an exposure time 
Membrane binding controls ordered self-assembly of animal septins

of $50 \mathrm{~ms}$. The microscope was controlled with MetaMorph 7.5 software (Molecular Devices, Sunnyvale, CA, USA). Images were processed (contrast enhancement and look-up table inversion) and analyzed (integrated intensity calculations) with Fiji software [112]. Fluorescence recovery after photobleaching was performed using a NikonA1 confocal microscope using a 100x oil immersion objective and an argon laser (Coherent, CA, USA) at excitation wavelengths of $488 \mathrm{~nm}$ for septins and $561 \mathrm{~nm}$ for rhodamine-PE lipids. The septins or the bilayer were first bleached by briefly $(1 \mathrm{~s})$ illuminating a circular region with a radius of $5 \mu \mathrm{m}$ using a high (70\%) output laser power. The fluorescence recovery was then measured for 5-7 minutes by illuminating the sample using a low (0.1-2\%) output laser power and $30 \mathrm{~ms}$ exposure time. For the first 30 seconds after the bleach, images were acquired at 1 frame per second. For the remaining acquisition time, images were acquired at a rate of 1 frame per 10 seconds.

\section{Scanning transmission electron microscopy of septin bundles}

To determine the morphology and mass per length (MPL) of septin bundles formed in solution, we performed scanning transmission electron microscopy (STEM) using tobacco mosaic virus (TMV) rods with a well-defined length $(300 \mathrm{~nm})$, width $(18 \mathrm{~nm})$ and MPL $(131 \mathrm{kDa} / \mathrm{nm})$ as an internal mass standard [113, 114]. Carbon or formvar+carbon coated copper grids (Ted Pella, Redding, CA, USA) were first incubated for $30 \mathrm{~s}$ with $3 \mu \mathrm{L}$ TMV (0.01-0.02 $\mathrm{mg} / \mathrm{ml}$ dispersion in phosphate-buffered saline), a kind gift from Dr. Jean-Luc Pellequer (Institut de Biologie Structurale, Grenoble, France), washed with ultrapure water, and blotted with filter paper. Next, $5 \mu \mathrm{L}$ of a solution of septins preassembled for $1 \mathrm{~h}$ at $20^{\circ} \mathrm{C}$ at a concentration between 50 and $500 \mathrm{nM}$ was deposited and left for $1 \mathrm{~min}$. Finally, the grids were washed with ultrapure water to remove excess salts and left to dry in air at $37^{\circ} \mathrm{C}$. Images of $3072 \times 2207$ pixels (16 bits) were acquired at different magnifications (from 15000 to $50000 \times$ ) and at an acceleration voltage of 10 to 20 $\mathrm{kV}$, a current of $100 \mathrm{pA}$, and a pixel dwell time of 3 to $5 \mu$ s on a FEI Verios 460 STEM microscope. The MPL was determined by analysis in Fiji [112] following a published procedure [114]. Briefly, we selected images that contained both TMV rods and septin bundles. For intensity calibration, boxes with a width of 25 to $45 \mathrm{~nm}$ (depending on the image resolution) and a length of $100 \mathrm{~nm}$ were drawn along the TMV rods, to obtain the protein signal, and on both sides of each rod, to obtain the background signal. The conversion factor from intensity (in counts) to mass (in $\mathrm{kDa}$ ) per pixel was determined from the integrated intensities of at least three TMV rods per image. A similar procedure was then used to obtain the MPL of septin bundles, drawing $100 \mathrm{~nm}$ long boxes over the bundles that encompassed their width (20 to $50 \mathrm{~nm}$ ), together with equal-sized boxes on either side of the bundle for background subtraction. The width of the septin bundles was measured by drawing a line in Fiji perpendicularly across septin bundles and estimating the distance between the edges by eye.

\section{Transmission electron microscopy of septins on lipid monolayers}

Samples for transmission electron microscopy (TEM) were prepared by incubating septin hexamers with lipid monolayers formed at an air-buffer interface. Teflon wells were filled with $20 \mu \mathrm{L}$ of a solution of 65 $\mathrm{nM}$ (fly) or $70 \mathrm{nM}$ (mammalian) septin hexamers in a $50 \mathrm{mM}$ Tris- $\mathrm{HCl}(\mathrm{pH}$ 8) buffer with $50 \mathrm{mM} \mathrm{KCl}$. A drop $(\sim 0.5 \mu \mathrm{L})$ of a $0.5 \mathrm{~g} / \mathrm{L}$ lipid solution in chloroform was deposited at the air-water interface in each well to form a lipid monolayer. The Teflon block was kept overnight at $4^{\circ} \mathrm{C}$ in a humid chamber, while stirring the solution in each well with a magnetic stirrer bar. Lipid monolayers with adsorbed septins were collected by briefly placing hydrophobic grids (Electron Microscopy Sciences, Hatfield, PA, USA) on the surface of the solutions with the carbon-coated side facing the solution. Grids with the collected monolayers facing 
Membrane binding controls ordered self-assembly of animal septins

up were stained at once with $2 \%$ uranyl formate (Electron Microscopy Sciences) in water by depositing 4 $\mu \mathrm{L}$ on the grid and simultaneously blotting the excess solution. Samples were imaged using a Tecnai Spirit electron microscope (Thermo Fischer) operated at an acceleration voltage of $120 \mathrm{~V}$. We repeated the experiments twice having each time 2 replicates.

\section{Cryo-electron microscopy of septins on large unilamellar lipid vesicles}

Samples for cryoEM were prepared by incubating septin hexamers with large unilamellar vesicles (LUVs). Lipid mixtures at a total lipid content of $50 \mu \mathrm{g}$ per sample in glass vials at the desired lipid ratios were dried with argon and left in vacuum for $2 \mathrm{~h}$ to remove chloroform. LUVs were formed by hydrating the lipids in high salt buffer at a concentration of $0.5 \mathrm{mg} / \mathrm{mL}(20 \mathrm{mM}$ imidazole $\mathrm{pH}$ 7.4, $1 \mathrm{mM}$ DTT, $300 \mathrm{mM}$ $\mathrm{KCl}$ and $2 \mathrm{mM} \mathrm{MgCl}$ ). The vesicles were next incubated for $30 \mathrm{~min}$ with $5 \mu \mathrm{g}$ of septins at a concentration of $1.6 \mu \mathrm{M}(0.5 \mathrm{~g} / \mathrm{L})$ in high salt septin storage buffer, corresponding to a lipid-to-protein weight ratio of 10:1. Meanwhile, lacey carbon coated grids (Electron Microscopy Science, EMS, France) were plasma treated for $30 \mathrm{~s}$ to hydrophilize the surface and remove any impurities. Vesicles diluted in septin polymerization buffer to a final concentration of $0.3 \mu \mathrm{M}(0.1 \mathrm{~g} / \mathrm{L})$ were applie [78]d to the grid straight after plasma treatment. Prior to the vitrification of the sample, $10 \mathrm{~nm}$ gold beads (EMS) were added in solution to be subsequently used as fiducial markers. Excess liquid was blotted and the samples were vitrified in liquid ethane using an automatic plunge freezer (EM PG, Leica, Wetzlar, Germany). Samples were imaged using a FEI Tecnai G2 microscope equipped with a LaB6 filament and a 4KX 4K CMOS camera F416 (TVIPS, Gauting, Germany) operated at an acceleration voltage of $200 \mathrm{kV}$ and a magnification of $50000 \times$. Data collection was carried out using the EMTool (TVIPS) software suite. Tilt series were acquired from -60 to 60 degrees using a saxton angular data collection scheme. Individual images were collected with 0.8 electrons per $\AA^{2}$ for a total dose of less than 70 electrons per $\AA^{2}$. IMOD software [115] was primarily used for data processing and alignment for individual images. The reconstructions were performed using either IMOD (Weighted back projection) or Tomo3D (SIRT) [116]. The segmentation of the volumes was performed manually using IMOD. We repeated the experiments three times.

\section{Atomic force microscopy of septins on supported lipid bilayers}

AFM samples were prepared on silicon substrates in order to approximate the conditions used for TIRF imaging and QCM-D measurements. Silicon wafers (Prime Silicon, Les Ulis, France) were cut into squares of $9 \times 9 \mathrm{~mm}^{2}$, rinsed with absolute ethanol, blow-dried with $\mathrm{N}_{2}$ gas, and treated with UV/ozone (Bioforce Nanoscience, Ames, IA) for 30 min to remove organic contaminants and render the surface hydrophilic. Each substrate was glued (Picodent Twinsil, Wipperfürth, Germany) on a 15-mm diameter metal disc (Agar Scientific) covered with a hydrophobic protector film (Bytac surface protection laminate; Sigma). An SLB was formed on the silicon wafer piece by incubating for $30 \mathrm{~min}$ with $100 \mu \mathrm{L}$ of a $100 \mu \mathrm{g} / \mathrm{mL}$ SUV solution diluted in septin polymerization buffer with an enhanced $\mathrm{KCl}$ concentration to promote SUV rupture (20 $\mathrm{mM}$ imidazole $\mathrm{pH} 7.4, \mathrm{pH} 7.4,1 \mathrm{mM} \mathrm{DTT}, 150 \mathrm{mM} \mathrm{KCl}$ and $2 \mathrm{mM} \mathrm{MgCl}$ ). Residual SUVs were washed off with $1 \mathrm{~mL}$ of septin polymerization buffer. The SLB was kept hydrated by leaving $100 \mu \mathrm{L}$ buffer on the wafer surface and $50 \mu \mathrm{L}$ of septin solution was added to reach a final concentration of either 12,24 or 60 $\mathrm{nM}$. After $15 \mathrm{~min}$ incubation at room temperature $\left(19^{\circ} \mathrm{C}\right)$, unbound protein was washed off with $1 \mathrm{~mL}$ of polymerization buffer. In most experiments, the samples were fixed for 1 minute with 1 wt-\% glutaraldehyde (GTA) in polymerization buffer and washed. Unfixed septins had a similar morphology as fixed septins but were difficult to image at high resolution because they were easily disrupted by the 
Membrane binding controls ordered self-assembly of animal septins

scanning AFM tip. Reproducibility across biological replicates was checked by performing experiments on 5 protein preps.

AFM images were acquired at room temperature $\left(23^{\circ} \mathrm{C}\right)$ with a Nanoscope Multimode 8 system (Bruker, CA, USA) on samples immersed in polymerization buffer. Images of various lateral dimensions ( 5 to $20 \mu \mathrm{m}$ ) at a resolution of $512 \times 512$ pixels and a scan rate of $0.8 \mathrm{~Hz}$ were recorded with silicon cantilevers (ScanAsyst-Fluid+; Bruker) with a nominal spring constant of $0.7 \mathrm{~N} / \mathrm{m}$ and a sharp pyramidal tip with a nominal radius of $2 \mathrm{~nm}$ and a maximal radius of $12 \mathrm{~nm}$. Images were recorded in Peak Force Tapping mode with a typical driving frequency of $2 \mathrm{kHz}$ and tapping amplitude of $50 \mathrm{~nm}$. Images were second-order flattened using open source software Gwyddion [117] to correct for sample tilt and curvature of the $x y$ piezo scanner. Analysis of septin thread heights and widths was performed by constructing height profiles (perpendicular to the thread axis, and averaging 5 pixels along the axis) in Gwyddion software. Heights were defined as the peak value above the flat membrane surface. Widths were measured at a height of 1 $\mathrm{nm}$ below peak height. Sections across narrow threads generally showed a single peak, but wider threads (> $30 \mathrm{~nm}$ in width) often showed multiple peaks. In the latter case, widths were measured at $1 \mathrm{~nm}$ below the height of the two outermost peaks. We recall that tip convolution effects lead to an enlarged effective width. Considering the expected tip shape, and the height at which the widths were measured, we expect tip convolution to entail a width overestimation by between 2 and $10 \mathrm{~nm}$.

\section{QCM-D measurements of septin-membrane binding}

To analyse the kinetics of septin binding and self-organisation on lipid membranes, we employed a surface analytical technique known as quartz crystal microbalance with dissipation monitoring (QCM-D). This is an acoustic technique that measures the adsorption of biomolecules onto the surface of a piezoelectric quartz crystal that is cyclically sheared at its acoustic resonance frequency by applying an alternating current [118]. The adsorption of molecules to the sensor surface results in a frequency shift $\Delta f$ that, to a first approximation, is proportional to the acoustic mass (including hydrodynamically coupled solvent) of the adsorbed film [119] and a dissipation shift $\Delta D$ that provides information on the mechanical properties of the film $[85,120]$.

We used silica-coated QCM-D sensors (QSX303, Biolin Scientific, Sweden) that were cleaned in an aqueous solution of $2 \%$ sodium dodecyl sulfate (30 min), rinsed with ultrapure water, blow-dried with $\mathrm{N}_{2}$ gas, and exposed to UV/ozone (BioForce Nanosciences, Ames, IA) for $30 \mathrm{~min}$. The sensors were then immediately mounted in the Q-Sense Flow Modules of the Q-Sense E4 system (Biolin Scientific) and experiments were started within 15 min. Experiments were performed at a working temperature of $23^{\circ} \mathrm{C}$. The instrument was operated in flow mode, meaning that sample solution was continuously perfused through the measurement chamber at a rate of $20 \mu \mathrm{L} / \mathrm{min}$ with a syringe pump (KD Scientific). We sequentially incubated the sensors with SUVs to form a bilayer and then septins, interspersed by rinsing steps. The surface was first equilibrated with vesicle buffer before flowing in the SUV suspension. The adsorption of the SUVs onto the sensor surface and the subsequent formation of a supported lipid bilayer (SLB) were monitored by measuring $\Delta f$ and $\Delta D$. The frequencies are measured in odd multiples (overtones) of the fundamental resonance frequency of the piezoelectric sensor crystal. In our experiments, the fundamental resonance frequency was $5 \mathrm{MHz}$ and data were recorded at overtones $i=$ 3 to 13 . For further analysis, we selected data obtained at the $7^{\text {th }}$ overtone, in view of its stable response across all measurements. Other overtones provided qualitatively similar information and are thus not shown. The frequency shifts were normalized according to $\Delta f=\Delta f_{7} / 7$. Once $\Delta f$ and $\Delta D$ reached stable 
Membrane binding controls ordered self-assembly of animal septins

values, indicating that the SLB was ready, the surface was rinsed with vesicle buffer to remove residual SUVs and then with septin polymerization buffer to equilibrate the ionic conditions. Next, the sensor was perfused with septins in polymerization buffer at the desired concentration (60 or $100 \mathrm{nM}$ ) for at least 60 min. Finally, we tested the reversibility of septin-membrane binding by rinsing the sensor with polymerization buffer. The experiments were performed at least in duplicate. Reproducibility across biological replicates was checked by performing experiments on 5 protein preps.

QCM-D measurements can provide information on the thickness of the adsorbed septin films. Provided that the septin layer is sufficiently uniform, dense and rigid, the Sauerbrey equation [119] relates the frequency shift to the areal mass density of the film, $m_{\mathrm{QCM}-\mathrm{D}}$, which equals the layer density $\rho_{\mathrm{QCM}-\mathrm{D}}$ times the layer thickness $d_{\mathrm{QCM}-\mathrm{D}}$ :

$$
\Delta f=-C^{1} \times m_{\mathrm{QCM}-\mathrm{D}}=-C^{-1} \times \rho_{\mathrm{QCM}-\mathrm{D}} \times d_{\mathrm{QCM}-\mathrm{D}}
$$

The mass sensitivity constant $C$ is $18 \mathrm{ng} / \mathrm{cm}^{2} / \mathrm{Hz}$ for QCM-D sensors with a fundamental resonance frequency of $5 \mathrm{MHz}$ and the mass density for protein films is $1.1 \mathrm{~g} / \mathrm{cm}^{3}$ to a good approximation [83]. For sufficiently dense and rigid $\left(\Delta D /-\Delta f \ll 0.4 \times 10^{-6} / \mathrm{Hz}\right)$ films, the film thickness $d_{\mathrm{QCM}-\mathrm{D}}$ is therefore proportional to $\Delta f$ with a proportionality constant of $6.1 \mathrm{~Hz} / \mathrm{nm}$.

\section{Acknowledgements}

We thank Marjolein Kuit-Vinkenoog for protein purification, Cristina Martinez Torres for discussions on the AFM data analysis and help with STEM imaging, Aditya lyer for help with STEM imaging, and Anders Aufderhorst-Roberts for help with AFM imaging. We also thank the Cell and Tissue Imaging (PICT-IBiSA), Institut Curie, member of the French National Research Infrastructure France-Biolmaging (ANR10-INBS04). AS and GHK gratefully acknowledge support by AMOLF, whose research program is part of the Netherlands Organisation for Scientific Research (NWO) and funding from the ERC (Starting Grant 335672; MINICELL). RPR and FB gratefully acknowledge support from the AFM facilities of the Molecular and Nanoscale Physics Group (University of Leeds) and funding from the ERC (Starting Grant 306435; JELLY) and the BBSRC (Equipment grant BB/R000174/1). MM and $A B$ gratefully acknowledge support by the Institut Curie, Institut Fresnel, and the Centre National de la Recherche Scientifique (CNRS) and funding from the Agence Nationale pour la Recherche (ANR grants ANR-13-JSV8-0002-01; SEPTIME and ANR-17CE13-0014; SEPTIMORF) and the Fondation ARC pour la recherche sur le cancer (grant PJA 20151203182).

\section{References}

1. Nishihama, R., M. Onishi, and J.R. Pringle, New insights into the phylogenetic distribution and evolutionary origins of the septins. Biol Chem, 2011. 392(8-9): p. 681-7.

2. Kartmann, B. and D. Roth, Novel roles for mammalian septins: from vesicle trafficking to oncogenesis. J Cell Sci, 2001. 114(Pt 5): p. 839-44.

3. Huang, Y.W., et al., Mammalian septins are required for phagosome formation. Mol Biol Cell, 2008. 19(4): p. 1717-26.

4. Dolat, L. and E.T. Spiliotis, Septins promote macropinosome maturation and traffic to the lysosome by facilitating membrane fusion. J Cell Biol, 2016. 214(5): p. 517-27.

5. Marquardt, J., X. Chen, and E. Bi, Architecture, remodeling, and functions of the septin cytoskeleton. Cytoskeleton (Hoboken), 2018. 

available under aCC-BY 4.0 International license.

Membrane binding controls ordered self-assembly of animal septins

966 6. Frazier, J., et al., Polymerization of purified yeast septins: evidence that organized filament arrays may not be required for septin function. J. Cell Biol., 1998. 143(3): p. 737-49.

968 7. Versele, M., et al., Protein-protein interactions governing septin heteropentamer assembly and septin filament organization in Saccharomyces cerevisiae. Mol Biol Cell, 2004. 15(10): p. 4568-83. Farkasovsky, M., et al., Nucleotide binding and filament assembly of recombinant yeast septin complexes. Biol Chem, 2005. 386(7): p. 643-56. p. 8274-9.

10. Khan, A., J. Newby, and A.S. Gladfelter, Control of septin filament flexibility and bundling by subunit composition and nucleotide interactions. Mol Biol Cell, 2018. 29(6): p. 702-712.

11. Byers, B. and L. Goetsch, A highly ordered ring of membrane-associated filaments in budding yeast. J Cell Biol, 1976. 69(3): p. 717-21.

12. Rodal, A.A., et al., Actin and septin ultrastructures at the budding yeast cell cortex. Mol Biol Cell, 2005. 16(1): p. 372-84.

13. Bertin, A., et al., Three-dimensional ultrastructure of the septin filament network in Saccharomyces cerevisiae Mol. Biol. Cell, 2012. 23(3): p. 423-32.

14. Longtine, M.S., et al., Septin-dependent assembly of a cell cycle-regulatory module in Saccharomyces cerevisiae. Mol Cell Biol, 2000. 20(11): p. 4049-61.

15. Finnigan, G.C., et al., Coordinate action of distinct sequence elements localizes checkpoint kinase Hsl1 to the septin collar at the bud neck in Saccharomyces cerevisiae. Mol Biol Cell, 2016. 27(14): p. 2213-33.

16. Tamborrini, D., et al., Recruitment of the mitotic exit network to yeast centrosomes couples septin displacement to actomyosin constriction. Nat Commun, 2018. 9(1): p. 4308.

17. Barral, Y., et al., Compartmentalization of the cell cortex by septins is required for maintenance of cell polarity in yeast. Mol Cell, 2000. 5(5): p. 841-51.

18. Takizawa, P.A., et al., Plasma membrane compartmentalization in yeast by messenger RNA transport and a septin diffusion barrier. Science, 2000. 290(5490): p. 341-4.

19. Clay, L., et al., A sphingolipid-dependent diffusion barrier confines ER stress to the yeast mother cell. Elife, 2014. 3: p. e01883.

20. Bridges, A.A. and A.S. Gladfelter, Septin Form and Function at the Cell Cortex. J Biol Chem, 2015. 290(28): p. 17173-80.

21. Spiliotis, E.T., Spatial effects - site-specific regulation of actin and microtubule organization by septin GTPases. J Cell Sci, 2018. 131(1).

1000

1001

1002

1003

1004

1005

1006

1007

1008

1009

1010

1011

1012

22. Cho, S.J., et al., Septin 6 regulates the cytoarchitecture of neurons through localization at dendritic branch points and bases of protrusions. Mol Cells, 2011. 32(1): p. 89-98.

23. Ewers, H., et al., A Septin-Dependent Diffusion Barrier at Dendritic Spine Necks. PLoS One, 2014. 9(12): p. e113916.

24. Palander, O., M. El-Zeiry, and W.S. Trimble, Uncovering the Roles of Septins in Cilia. Front Cell Dev Biol, 2017. 5: p. 36.

25. Tooley, A., et al., Amoeboid T lymphocytes require the septin cytoskeleton for cortical integrity and persistent motility. Nat. Cell Biol., 2009. 11(1): p. 17-26.

26. Mostowy, S., et al., A role for septins in the interaction between the Listeria monocytogenes invasion protein InIB and the Met receptor. Biophys. J., 2011. 100(8): p. 1949-59.

27. Gilden, J., et al., The septin cytoskeleton facilitates membrane retraction during motility and blebbing J. Cell Biol., 2012. 196(1): p. 103-114.

28. Shindo, A. and J.B. Wallingford, PCP and septins compartmentalize cortical actomyosin to direct collective cell movement. Science, 2014. 343(6171): p. 649-52. 
Membrane binding controls ordered self-assembly of animal septins

1014

1015

1016

1017

1018

1019

1020

1021

1022

1023

1024

1025

1026

1027

1028

1029

1030

1031

1032

1033

1034

1035

1036

1037

1038

1039

1040

1041

1042

1043

1044

1045

1046

1047

1048

1049

1050

1051

1052

1053

1054

1055

1056

1057

1058

1059

1060

1061

29. Park, T.J., S.K. Kim, and J.B. Wallingford, The planar cell polarity effector protein Wdpcp (Fritz) controls epithelial cell cortex dynamics via septins and actomyosin. Biochem Biophys Res Commun, 2015. 456(2): p. 562-6.

30. Kim, J. and J.A. Cooper, Septins regulate junctional integrity of endothelial monolayers. Mol Biol Cell, 2018. 29(13): p. 1693-1703.

31. Founounou, N., N. Loyer, and R. Le Borgne, Septins regulate the contractility of the actomyosin ring to enable adherens junction remodeling during cytokinesis of epithelial cells. Dev Cell, 2013. 24(3): p. 242-55.

32. Spiliotis, E., M. Kinoshita, and W. Nelson, A mitotic septin scaffold required for mammalian chromosome congression and segregation Science, 2005. 307(5716): p. 1781-85.

33. Kim, M., et al., SEPT9 occupies the terminal positions in septin octamers and mediates polymerization-dependent functions in abscission. J. Cell Biol., 2011. 195(5): p. 815-826.

34. Renshaw, M.J., et al., Anillin-dependent organization of septin filaments promotes intercellular bridge elongation and Chmp4B targeting to the abscission site. Open Biol, 2014. 4: p. 130190.

35. Gilden, J. and M. Krummel, Control of cortical rigidity by the cytoskeleton: emerging roles for septins Cytoskeleton, 2010. 67(8): p. 477-486.

36. Hagiwara, A., et al., Submembranous Septins as Relatively Stable Components of Actin-Based Membrane Skeleton Cytoskeleton, 2011. 68(9): p. 512-525.

37. Zhang, J., et al., Phosphatidylinositol polyphosphate binding to the mammalian septin H5 is modulated by GTP. Curr. Biol., 1999. 9(24): p. 1458-67.

38. Tanaka, T., M. Kinoshita, and K. Takiguchi, Septin-mediated uniform bracing of phospholipid membranes. Curr. Biol., 2009. 19(2): p. 140-5.

39. Bridges, A.A., et al., Micron-scale plasma membrane curvature is recognized by the septin cytoskeleton. J Cell Biol, 2016. 213(1): p. 23-32.

40. Yamada, S., et al., Septin Interferes with the Temperature-Dependent Domain Formation and Disappearance of Lipid Bilayer Membranes. Langmuir, 2016. 32(48): p. 12823-12832.

41. Mavrakis, M., et al., Septins promote F-actin ring formation by crosslinking actin filaments into curved bundles. Nat Cell Biol, 2014. 16(4): p. 322-34.

42. Dolat, L., et al., Septins promote stress fiber-mediated maturation of focal adhesions and renal epithelial motility. J Cell Biol, 2014. 207(2): p. 225-35.

43. Smith, C., et al., Septin 9 Exhibits Polymorphic Binding to F-Actin and Inhibits Myosin and Cofilin Activity. J Mol Biol, 2015. 427(20): p. 3273-3284.

44. Kinoshita, M., et al., Self- and actin-templated assembly of mammalian septins. Dev. Cell, 2002. 6(791-802).

45. Joo, E., M. Surka, and W. Trimble, Mammalian SEPT2 is required for scaffolding nonmuscle myosin Il and its kinases. Dev. Cell, 2007. 13(5): p. 677-90.

46. Kinoshita, M., et al., Nedd5, a mammalian septin, is a novel cytoskeletal component interacting with actin-based structures. Genes Dev, 1997. 11(12): p. 1535-47.

47. Sellin, M., et al., Microtubules support a disk-like septin arrangement at the plasma membrane of mammalian cells Mol. Biol. Cell, 2011. 22(23): p. 4588-4601.

48. Mendoza, M., A. Hyman, and M. Glotzer, GTP binding induces filament assembly of a recombinant septin. Curr. Biol., 2002. 12(21): p. 1858-63.

49. John, C., et al., The Caenorhabditis elegans septin complex is nonpolar. EMBO J., 2007. 26(14): p. 3296-307.

50. Field, C., et al., A purified Drosophila septin complex forms filaments and exhibits GTPase activity. J. Cell Biol., 1996. 133(3): p. 605-16.

51. Huijbregts, R.P., et al., Drosophila Orc6 facilitates GTPase activity and filament formation of the septin complex. Mol Biol Cell, 2009. 20(1): p. 270-81. 
Membrane binding controls ordered self-assembly of animal septins

1062

1063

1064

1065

1066

1067

1068

1069

1070

1071

1072

1073

1074

1075

1076

1077

1078

1079

1080

1081

1082

1083

1084

1085

1086

1087

1088

1089

1090

1091

1092

1093

1094

1095

1096

1097

1098

1099

1100

1101

1102

1103

1104

1105

1106

1107

1108

52. Garcia III, G., et al., Subunit-dependent modulation of septin assembly: Budding yeast septin Shs1 promotes ring and gauze formation J. Cell Biol., 2011. 195(6): p. 993-1004.

53. Leventis, P.A. and S. Grinstein, The distribution and function of phosphatidylserine in cellular membranes. Annu Rev Biophys, 2010. 39: p. 407-27.

54. Carvalho, M., et al., Effects of diet and development on the Drosophila lipidome. Mol Syst Biol, 2012. 8: p. 600.

55. Guan, X.L., et al., Biochemical membrane lipidomics during Drosophila development. Dev Cell, 2013. 24(1): p. 98-111.

56. Jones, H.E., et al., Lipid composition of subcellular membranes from larvae and prepupae of Drosophila melanogaster. Lipids, 1992. 27(12): p. 984-7.

57. Bertin, A., et al., Phosphatidylinositol-4,5-bisphosphate promotes budding yeast septin filament assembly and organization. J. Mol. Biol., 2010. 404(4): p. 711-31.

58. Beber, A., et al., Septin-based readout of PI(4,5)P2 incorporation into membranes of giant unilamellar vesicles. Cytoskeleton (Hoboken), 2018.

59. Brill, J.A., S. Yildirim, and L. Fabian, Phosphoinositide signaling in sperm development. Semin Cell Dev Biol, 2016. 59: p. 2-9.

60. Goldbach, P., et al., Stabilization of the actomyosin ring enables spermatocyte cytokinesis in Drosophil. Mol. Biol. Cell, 2010. 21(9): p. 1482-93.

61. Neufeld, T. and G. Rubin, The Drosophila peanut gene is required for cytokinesis and encodes a protein similar to yeast putative bud neck filament proteins. Cell, 1994. 77(3): p. 371-9.

62. Fares, H., M. Peifer, and J. Pringle, Localization and possible functions of Drosophila septins. Mol. Biol. Cell, 1995. 6(12): p. 1843-59.

63. Adam, J., J. Pringle, and M. Peifer, Evidence for functional differentiation among Drosophila septins in cytokinesis and cellularization. Mol. Biol. Cell, 2000. 11(9): p. 3123-35.

64. Cao, L., et al., Phylogenetic and evolutionary analysis of the septin protein family in metazoan. FEBS Lett, 2007. 581(28): p. 5526-32.

65. Pan, F., R.L. Malmberg, and M. Momany, Analysis of septins across kingdoms reveals orthology and new motifs. BMC Evol Biol, 2007. 7: p. 103.

66. Mendonca, D.C., et al., A revised order of subunits in mammalian septin complexes. Cytoskeleton (Hoboken), 2019. 76(9-10): p. 457-466.

67. Soroor, F., et al., Revised subunit order of mammalian septin complexes explains their in vitro polymerization properties. bioRxiv:doi.org/10.1101/569871, 2019.

68. Marques, I., et al., Septin C-Terminal Domain Interactions: Implications for Filament Stability and Assembly Cell Biochem. Biophys., 2012. 62(2): p. 317-328.

69. Sirajuddin, M., et al., Structural insight into filament formation by mammalian septins. Nature, 2007. 449(7160): p. 311-5.

70. Mavrakis, M., F.C. Tsai, and G.H. Koenderink, Purification of recombinant human and Drosophila septin hexamers for TIRF assays of actin-septin filament assembly. Methods Cell Biol, 2016. 136: p. $199-220$.

71. Sousa, A.A. and R.D. Leapman, Development and application of STEM for the biological sciences. Ultramicroscopy, 2012. 123: p. 38-49.

72. Blin, G., et al., Quantitative analysis of the binding of ezrin to large unilamellar vesicles containing phosphatidylinositol 4,5 bisphosphate. Biophys J, 2008. 94(3): p. 1021-33.

73. Kooijman, E.E., et al., lonization properties of phosphatidylinositol polyphosphates in mixed model membranes. Biochemistry, 2009. 48(40): p. 9360-71.

74. Toner, M., et al., Adsorption of cations to phosphatidylinositol 4,5-bisphosphate. Biochemistry, 1988. 27(19): p. 7435-43. 
Membrane binding controls ordered self-assembly of animal septins

75. Graber, Z.T., et al., Phosphatidylinositol-4,5-bisphosphate ionization and domain formation in the presence of lipids with hydrogen bond donor capabilities. Chem Phys Lipids, 2012. 165(6): p. 696704.

76. McLaughlin, S. and D. Murray, Plasma membrane phosphoinositide organization by protein electrostatics. Nature, 2005. 438(7068): p. 605-11.

77. Yeung, T., et al., Membrane phosphatidylserine regulates surface charge and protein localization. Science, 2008. 319(5860): p. 210-3.

78. Beber, A., et al., Membrane reshaping by micrometric curvature sensitive septin filaments. Nat Commun, 2019. 10(1): p. 420.

79. Cannon, K.S., et al., An amphipathic helix enables septins to sense micron-scale membrane curvature. J. Cell Biol., 2019. DOI: 10.1083/jcb.201807211.

80. Richter, R., A. Mukhopadhyay, and A. Brisson, Pathways of lipid vesicle deposition on solid surfaces: a combined QCM-D and AFM study. Biophys J, 2003. 85(5): p. 3035-47.

81. Richter, R.P., N. Maury, and A.R. Brisson, On the effect of the solid support on the interleaflet distribution of lipids in supported lipid bilayers. Langmuir, 2005. 21(1): p. 299-304.

82. Zhang, R., et al., Interplay of structure, elasticity, and dynamics in actin-based nematic materials. Proc Natl Acad Sci U S A, 2018. 115(2): p. E124-e133.

83. Reviakine, I., D. Johannsmann, and R.P. Richter, Hearing what you cannot see and visualizing what you hear: interpreting quartz crystal microbalance data from solvated interfaces. Anal Chem, 2011. 83(23): p. 8838-48.

84. Richter, R., R. Berat, and A. Brisson, Formation of solid-supported lipid bilayers: An integrated view. Langmuir, 2006. 22(8): p. 3497-3505.

85. Johannsmann, D., I. Reviakine, and R.P. Richter, Dissipation in films of adsorbed nanospheres studied by quartz crystal microbalance (QCM). Anal Chem, 2009. 81(19): p. 8167-76.

86. Tsortos, A., G. Papadakis, and E. Gizeli, Shear acoustic wave biosensor for detecting DNA intrinsic viscosity and conformation: a study with QCM-D. Biosens Bioelectron, 2008. 24(4): p. 842-7.

87. Thakar, D., et al., A quartz crystal microbalance method to study the terminal functionalization of glycosaminoglycans. Chem Commun (Camb), 2014. 50(96): p. 15148-51.

88. Bridges, A.A., et al., Septin assemblies form by diffusion-driven annealing on membranes. Proc Natl Acad Sci U S A, 2014. 111(6): p. 2146-51.

89. Oling, F., W. Bergsma-Schutter, and A. Brisson, Trimers, dimers of trimers, and trimers of trimers are common building blocks of annexin a5 two-dimensional crystals. J Struct Biol, 2001. 133(1): p. 55-63.

90. Ong, K., et al., Architecture and dynamic remodelling of the septin cytoskeleton during the cell cycle. Nat Commun, 2014. 5: p. 5698.

91. Booth, E.A. and J. Thorner, A FRET-based method for monitoring septin polymerization and binding of septin-associated proteins. Methods Cell Biol., 2016. 136: p. 35-56.

92. Casamayor, A. and M. Snyder, Molecular dissection of a yeast septin: distinct domains are required for septin interaction, localization, and function. Mol. Cell Biol., 2003. 23(8): p. 2762-77.

93. Onishi, M., et al., Role of septins in the orientation of forespore membrane extension during sporulation in fission yeast. Mol Cell Biol, 2010. 30(8): p. 2057-74.

94. Lee, K.I., W. Im, and R.W. Pastor, Langevin dynamics simulations of charged model phosphatidylinositol lipids in the presence of diffusion barriers: toward an atomic level understanding of corralling of PIP2 by protein fences in biological membranes. BMC Biophys, 2014. 7: p. 13.

95. Castro, D., et al., A complete compendium of crystal structures for the human SEPT3 subgroup reveals functional plasticity at a specific septin interface. IUCrJ, 2020. 7(Pt 3): p. 462-479. 
Membrane binding controls ordered self-assembly of animal septins

1156

1157

1158

1159

1160

1161

1162

1163

1164

1165

1166

1167

1168

1169

1170

1171

1172

1173

1174

1175

1176

1177

1178

1179

1180

1181

1182

1183

1184

1185

1186

1187

1188

1189

1190

1191

1192

1193

1194

1195

1196

1197

1198

1199

1200

1201

1202

96. Omrane, M., et al., Septin 9 has Two Polybasic Domains Critical to Septin Filament Assembly and Golgi Integrity. iScience, 2019. 13: p. 138-153.

97. Woolfson, D.N., Coiled-Coil Design: Updated and Upgraded. Subcell Biochem, 2017. 82: p. 35-61.

98. Lupas, A.N., J. Bassler, and S. Dunin-Horkawicz, The Structure and Topology of $\alpha$-Helical Coiled Coils. Subcell Biochem, 2017. 82: p. 95-129.

99. Bovellan, M., et al., Cellular control of cortical actin nucleation. Curr Biol, 2014. 24(14): p. 16281635.

100. Fritzsche, M., et al., Actin kinetics shapes cortical network structure and mechanics. Sci Adv, 2016. 2(4): p. e1501337.

101. Fischer-Friedrich, E., et al., Rheology of the Active Cell Cortex in Mitosis. Biophys J, 2016. 111(3): p. 589-600.

102. Clement, R., et al., Viscoelastic Dissipation Stabilizes Cell Shape Changes during Tissue Morphogenesis. Curr Biol, 2017. 27(20): p. 3132-3142.e4.

103. Kanchanawong, P., et al., Nanoscale architecture of integrin-based cell adhesions. Nature, 2010. 468(7323): p. 580-4.

104. Birdsall, B., et al., Correction for light absorption in fluorescence studies of protein-ligand interactions. Anal Biochem, 1983. 132(2): p. 353-61.

105. Gasteiger, E., et al., ExPASy: The proteomics server for in-depth protein knowledge and analysis. Nucleic Acids Res, 2003. 31(13): p. 3784-8.

106. Lupas, A., M. Van Dyke, and J. Stock, Predicting coiled coils from protein sequences. Science, 1991. 252(5009): p. 1162-4.

107. Kelley, L.A., et al., The Phyre2 web portal for protein modeling, prediction and analysis. Nat Protoc, 2015. 10(6): p. 845-58.

108. Buchan, D.W.A. and D.T. Jones, The PSIPRED Protein Analysis Workbench: 20 years on. Nucleic Acids Res, 2019. 47(W1): p. W402-w407.

109. Braunger, J.A., et al., Solid supported membranes doped with PIP2: influence of ionic strength and pH on bilayer formation and membrane organization. Langmuir, 2013. 29(46): p. 14204-13.

110. Rasnik, I., S.A. McKinney, and T. Ha, Nonblinking and long-lasting single-molecule fluorescence imaging. Nat Methods, 2006. 3(11): p. 891-3.

111. Aitken, C.E., R.A. Marshall, and J.D. Puglisi, An oxygen scavenging system for improvement of dye stability in single-molecule fluorescence experiments. Biophys J, 2008. 94(5): p. 1826-35.

112. Schindelin, J., et al., Fiji: an open-source platform for biological-image analysis. Nat Methods, 2012. 9(7): p. 676-82.

113. Freeman, R. and K.R. Leonard, Comparative mass measurement of biological macromolecules by scanning transmission electron microscopy. J Microsc, 1981. 122(Pt 3): p. 275-86.

114. Sousa, A.A. and R.D. Leapman, Mass mapping of amyloid fibrils in the electron microscope using STEM imaging. Methods Mol Biol, 2013. 950: p. 195-207.

115. Kremer, J.R., D.N. Mastronarde, and J.R. McIntosh, Computer visualization of three-dimensional image data using IMOD. J Struct Biol, 1996. 116(1): p. 71-6.

116. Agulleiro, J.I. and J.J. Fernandez, Fast tomographic reconstruction on multicore computers. Bioinformatics, 2011. 27(4): p. 582-3.

117. Nečas, D. and P. Klapetek, Gwyddion: An open-source software for SPM data analysis. Central European Journal of Physics, 2012. 10(1): p. 181-188.

118. Ward, M.D. and D.A. Buttry, In situ interfacial mass detection with piezoelectric transducers. Science, 1990. 249(4972): p. 1000-7.

119. Sauerbrey, G., Verwendung von Schwingquarzen zur Wägung dünner Schichten und zur Mikrowägung. Zeitschrift für Physik, 1959. 155(2): p. 206-222. 
bioRxiv preprint doi: https://doi.org/10.1101/2020.09.22.307918; this version posted September 23, 2020. The copyright holder for this preprint (which was not certified by peer review) is the author/funder, who has granted bioRxiv a license to display the preprint in perpetuity. It is made available under aCC-BY 4.0 International license.

Membrane binding controls ordered self-assembly of animal septins

1203

1204

1205

120. Eisele, N.B., et al., Viscoelasticity of thin biomolecular films: a case study on nucleoporin phenylalanine-glycine repeats grafted to a histidine-tag capturing QCM-D sensor. Biomacromolecules, 2012. 13(8): p. 2322-32.

1206 